\title{
Integrative analysis of TNFRSF6B as a potential therapeutic target for pancreatic cancer
}

\author{
Chen Zhang ${ }^{1 \#}$, Haoran $\mathrm{Li}^{1 \#}$, Yujie Huang ${ }^{1}$, Yuchen Tang ${ }^{1}$, Jie Wang ${ }^{1}$, Yinxiang Cheng ${ }^{1}$, Yijun Wei ${ }^{1}$, \\ Dongming Zhu ${ }^{1}$, Zhifei $\mathrm{Cao}^{2}$, Jian Zhou ${ }^{1}$
}

${ }^{1}$ Department of General Surgery, The First Affiliated Hospital of Soochow University, Suzhou, China; ${ }^{2}$ Department of Pathology, The Second Affiliated Hospital of Soochow University, Suzhou, China

Contributions: (I) Conception and design: J Zhou, Z Cao, C Zhang, H Li; (II) Administrative support: J Zhou, D Zhu; (III) Provision of study materials or patients: J Zhou, C Zhang; (IV) Collection and assembly of data: Y Huang, Y Tang, J Wang; (V) Data analysis and interpretation: C Zhang, H Li, Y Cheng, Y Wei; (VI) Manuscript writing: All authors; (VII) Final approval of manuscript: All authors.

\#These authors contributed equally to this work.

Correspondence to: Jian Zhou. Department of General Surgery, The First Affiliated Hospital of Soochow University, 188 Shizi Street, Suzhou 215006 , China. Email: zhoujian06@suda.edu.cn; Zhifei Cao. Department of Pathology, The Second Affiliated Hospital of Soochow University, Suzhou 215004, China. Email: hunancao@163.com.

Background: Pancreatic cancer is one of the most lethal malignant tumors worldwide with poor outcomes. Previous studies have shown that tumor necrosis factor receptor superfamily member 6b (TNFRSF6B) plays an important role in cancer progression and immunosuppression. However, the mechanisms by which TNFRSF6B influence pancreatic cancer, and the regulatory networks involved remain to be further studied.

Methods: This study analyzed the mRNA information and clinical data of patients from The Cancer Genome Atlas (TCGA) and the ONCOMINE databases. The gene co-expression data regarding TNFRSF6B was obtained from the c-BioPortal and used to explore the functional network of TNFRSF6B in pancreatic cancer, as well as its function in tumor immunity. Short hairpin (sh) RNA knock-down experiments were performed to examine the functional roles of TNFRSF6B in pancreatic cancer cell lines.

Results: The expression of TNFRSF6B was elevated in pancreatic cancer tissues compared to normal pancreatic tissues, and its high expression was associated with poor prognosis of patients with pancreatic cancer. TNFRSF6B was found to be widely involved in cell cycle processes, apoptosis, apoptosis signaling pathways, immune responses, and responses to interferon. Knock-down of TNFRSF6B expression inhibited pancreatic cancer cell proliferation and invasion in vitro. Moreover, carcinoembryonic antigen-related cell adhesion molecule 1 (CEACAM1) was found to be co-expressed with TNFRSF6B, and there was a positive correlation between these molecules in pancreatic cancer cells.

Conclusions: This report suggested that TNFRSF6B has a critical role in the progression and metastasis of pancreatic cancer. These findings provide novel insights into the role of TNFRSF6B in the functional network of pancreatic cancer, and suggest that TNFRSF6B may be a potential therapeutic target.

Keywords: TNFRSF6B; pancreatic cancer; functional networks; tumor immunity

Submitted May 10, 2021. Accepted for publication Jun 23, 2021.

doi: 10.21037/jgo-21-303

View this article at: https://dx.doi.org/10.21037/jgo-21-303

\section{Introduction}

Pancreatic cancer is one of the most common gastrointestinal malignant tumors in the world. It accounts for $5 \%$ of cancer-related deaths and is recognized as the fourth leading cause of cancer-related mortality (1). This is largely due to late diagnosis, low resection rates, and high drug resistance to chemotherapy (2). Diagnosis of the disease is exceedingly difficult due to a lack of 
early symptoms and the malignancy can spread rapidly to surrounding organs. At diagnosis, more than $70 \%$ of patients present with advance stage disease or distant metastases, making surgical treatment difficult. Invasion and metastasis are the most important characteristics of pancreatic cancer, and thus, there is an urgent need to understand the molecular mechanisms involved in these processes.

In recent years, secretory proteins have increasingly gained prominence in the fields of tumor invasion, metastasis, and drug resistance $(3,4)$. Pancreatic cancer cells can produce a large number of secretory proteins, which promote extracellular matrix (ECM) degradation and tumor metastasis (5). These tumor-specific proteins play an important role in many cellular functions, including signal transduction and interaction with plasma membrane (6,7). TNFRSF6B is a soluble secretory protein which lacks the transmembrane structure in its amino acid sequence (8). The overexpression of TNFRSF6B protein may block apoptosis and immune monitoring by competitively binding with first apoptosis signal ligand (FasL) (9), tumor necrosis factor-like cytokine 1A (TL1A) (10), or lymphotoxin analogue (LIGHT, also known as TNFSF14) (11). Therefore, TNFRSF6B is also known as decoy receptor $3(D c R 3)$. Previous literature demonstrated that TNFRSF6B is highly expressed in human tumors such as lung cancer $(12,13)$, gastric cancer (14), pancreatic cancer $(15,16)$, and colon cancer $(17,18)$, and it has been reported that overexpression of DcR3 is correlated with shortened total survival time of cancer patients (19). Our previous study found that TNFRSF6B-mediated signal transduction pathways play an important role in regulating proliferation, apoptosis, migration, and invasion of pancreatic cancer cells (20). TNFRSF6B is the key node in the regulatory network for the occurrence and development of pancreatic cancer, however, its regulatory relationship is complex, and the precise mechanisms are still unclear.

This report investigated TNFRSF $6 B$ expression in pancreatic cancer using databases including The Cancer Genome Atlas (TCGA), the Gene Expression Profiling Interactive Analysis (GEPIA), and ONCOMINE. Through multidimensional analyses, the functional networks and potential relationships of TNFRSF $6 B$ in pancreatic cancer, and its role in tumor immunity were evaluated. This study provides novel insights into potential therapeutic targets and strategies for the treatment of pancreatic cancer. We present the following article in accordance with the MDAR checklist (available at https:// dx.doi.org/10.21037/jgo-21-303).

\section{Methods}

\section{Acquisition of patients and data}

All mRNA information and clinical data of patients were obtained from the University of California, Santa Cruz (UCSC) website (https://xena.ucsc.edu/). The cBioPortal website (https://www.cbioportal.org/) was search to obtain the co-expression gene data related to TNFRSF6B. This study was conducted in accordance with the Declaration of Helsinki (as revised in 2013). Since all data were extracted from publicly available databases, approval by the ethics committee and patient consent were waived.

\section{TCGA database analysis}

To provide comprehensive analysis of cancer genome profiles, the TCGA applies high-throughput technologies based on microarrays and next-generation sequencing methods. The research network structure includes many centers utilizing different platforms to provide global information related to cancer genomics.

\section{ONCOMINE database analysis}

The levels of TNFRSF6B gene expression in various types of cancers were identified in the ONCOMINE database (https://www.oncomine.org/resource/login.html) (21). The threshold applied was a $\mathrm{P}$ value of $1 \mathrm{E}-4$, fold change of 2 , and gene ranking of $10 \%$.

\section{GEPIA database analysis}

The Gene Expression Profiling Interactive Analysis (GEPIA) database (http://gepia.cancer-pku.cn/) is an interactive database that includes 9,736 tumors and 8,587 normal samples from the TCGA and the Genotype-Tissue Expression (GTEx) projects (22). GEPIA was used to generate survival curves based on gene expression with the log-rank test.

\section{TIMER database analysis}

TIMER (https://cistrome.shinyapps.io/timer/) is a comprehensive resource for systematic analysis of immune infiltrates across diverse cancer types from the TCGA, 
which includes 10,897 samples across 32 cancer types (23).

The correlation between TNFRSF $6 B$ expression and the abundance of immune infiltrates, including macrophages, neutrophils, dendritic cells, B cells, CD4+ T cells, and CD8+ T cells was analyzed.

\section{GeneMANIA analysis}

GeneMANIA (http://www.genemania.org) is a flexible, user-friendly web interface for constructing protein-protein interaction (PPI) networks, generating hypotheses related to gene function, analyzing gene lists, and prioritizing genes for functional assays (24). The website can set the source of the edge of the network, and it features several bioinformatics methods including physical interaction, gene co-expression, gene co-location, gene enrichment analysis, and website prediction. GeneMANIA was used to visualize the gene networks.

\section{Construction of the co-expression network}

The co-expression gene data of TNFRSF6B was downloaded from c-BioPortal and used to identify genes with Spearman's Correlation greater than 0.4 and less than -0.4. A total of 1,143 genes were extracted from 168 patients with complete clinical data. To further analyze the relationship between these co-expression genes, the R packet WGCNA (weighted gene co-expression network analysis) was used to construct a co-expression network. The "pickSoft Threshold" function was used to select the best soft threshold, and finally the soft threshold was determined to be 11 . The minimum number of genes in the module was defined as 30 .

\section{Identification of the bub module and functional annotation}

The correlation between ME (module eigengenes) and the clinical information of each module was analyzed. The modules closely related to prognosis were selected. The relationship between different modules and age, gender, T stage, $\mathrm{N}$ stage, TNM stage, overall survival (OS) time, disease-specific survival (DSS) time, progression-free survival (PFI) time, and disease-free interval (DFI) was studied. At the same time, an in-depth analysis of the genes in the key modules was performed. CLUEGO was used for KEGG (Kyoto Encyclopedia of Genes and Genomes) and GO (Gene Ontology) enrichment analysis of genes in key modules to explore possible pathways and molecular mechanisms. GeneMANIA (https://genemania.org/) was used to constructed the PPI network of 10 hub genes and TNFRSFGB.

\section{Immune cells and immune checkpoint genes}

Single-sample gene set enrichment analysis (SsGESA) was performed to estimate the status of immune cells in patients with pancreatic cancer. The Tumor Immune Estimation Resource (TIMER) website (http://timer.cistrome. org/) was used to evaluate the infiltration of six different kinds of immune cells in patients with pancreatic cancer. Furthermore, the expression of immune checkpoint genes in the TCGA cohort was investigated.

\section{Short hairpin (sh) RNA lentiviral plasmids construction}

A shRNA sequence targeting TNFRSF6B (GenBank: NM003823) (5'-CGCTGGTTTCTGCTTGGAGCAC-3') was subcloned into a lentiviral vector (sh-TNFRSF6B), and a lentiviral vector containing a random sequence was used as a negative control (sh-NC). The viral supernatant was added at a multiplicity of infection (MOI) of 5 per well with $5 \mathrm{mg} / \mathrm{mL}$ polybrene.

\section{Microarray and data analysis}

Sh-TNFRSF6B cells $(\mathrm{n}=3)$ and sh-NC cells $(\mathrm{n}=3)$ were collected for total RNA extraction. The Affymetrix gene chip array (Thermo Fisher Science, Massachusetts, USA) was used to detect the genome-wide expression changes in the extracted RNA samples according to the manufacturer's instructions. Differential genes were screened according to $\mathrm{P}$ value $<0.05$ and absolute fold change $>2$. The ingenuity pathway analysis (IPA) software was used to classify differentially expressed genes into specific biological functions and pathways related to TNFRSF6B genes.

\section{Clone formation assay}

Sh-TNFRSF6B and sh-NC cells were seeded into 6-well plates in a density of 500 cells/well. Then they were placed into the constant temperature chamber in an atmosphere of $5 \% \mathrm{CO}_{2}$ at $37{ }^{\circ} \mathrm{C}$ for 2 weeks incubation with media changes every 2 or 3 days. Cells were then fixed with $4 \%$ paraformaldehyde and stained with $1 \%$ crystal violet. Cell colonies containing at least 20 cells were counted using an inverted microscope at $40 \times$ magnification. The experiments were performed three times. 


\section{Cell invasion assay}

Cell migration assays were performed using $8-\mu \mathrm{m}$ Transwell ${ }^{\circledR}$ cell culture chambers (Corning, NY, USA, pore size of $8 \mu \mathrm{m})$. Cells $\left(5 \times 10^{5}\right.$ cells $\left./ \mathrm{mL}\right)$ were resuspended in serum-free Dulbecco's Modified Eagle Medium (DMEM) and seeded into the upper well of the Transwell chamber. The well below the Transwell chamber was filled with DMEM supplemented with $10 \%$ fetal bovine serum (FBS). After incubation for 48 hours at $37^{\circ} \mathrm{C}$, cells that had migrated to the bottom surface of the chamber were fixed with methanol and stained with $0.1 \%$ crystal violet. The membranes containing the cells were then imaged under light microscopy and the number of cells from at least five random fields of view were counted.

\section{Western blot}

Total proteins were extracted from cells with lysis buffer. Protein samples were separated using $10 \%$ sodium dodecyl sulphate-polyacrylamide gel electrophoresis (SDS-PAGE) and transferred to poly vinylidene fluoride (PVDF) filter membranes (Roche, Switzerland). After blocking in phosphate buffered saline (PBS) containing $0.05 \%$ Tween-20 and 5\% non-fat milk powder, the membranes were incubated with primary antibodies at $4{ }^{\circ} \mathrm{C}$ overnight (DcR3 and CEACAM1 at 1:1,000 dilution; and glyceraldehyde 3-phosphate dehydrogenase [GAPDH] at 1:5,000 dilution). Membranes were then washed and incubated at room temperature for $2 \mathrm{~h}$ with a secondary horseradish peroxidase-conjugated goat anti-mouse IgG $(1: 5,000)$ or goat anti-rabbit IgG $(1: 5,000)$. The protein bands on the membranes were detected using an enhanced chemiluminescence (ECL) detection system (FDbio, China). All experiments were performed according to the manufacturer's instructions.

\section{Co-immunoprecipitation (CO-IP)}

Cells were washed with PBS, centrifuged at $15,000 \mathrm{~g}$ at $4{ }^{\circ} \mathrm{C}$ for 10 minutes, and the supernatant was collected and incubated with the primary antibody or mouse IgG control at $4{ }^{\circ} \mathrm{C}$ for 4 hours. Subsequently, $20 \mu \mathrm{L}$ of pre-washed protein $\mathrm{A} / \mathrm{G}$ sepharose beads was incubated with $400 \mu \mathrm{g}$ of sample overnight at $4{ }^{\circ} \mathrm{C}$. After incubation, the immune complex was washed 3 times with lysis buffer, the protein was collected after centrifugation, and western blot analysis was performed.

\section{Immunofluorescence (IF) staining}

Cells were cultured and fixed on glass slides. After reaching confluence, cells were incubated with $0.1 \%$ Triton X-100 solution in PBS for 10 minutes to permeabilize the cells. Cells were then incubated with primary antibodies against $\beta$-catenin, followed by addition of the secondary antibodies. The slides were dyed with DAPI (4,6-diamidino-2phenylindole) and examined with confocal microscopy (Nikon Corporation, Tokyo, Japan).

\section{EdU (5-Ethynyl-2'-deoxyuridine) assay}

The Cell-Light EdU DNA Cell Proliferation Kit (RiboBio, Guangzhou, China) was used to perform proliferation assays according to the manufacturer's protocol. Briefly, cells were incubated with EdU for 2 hours, followed by fixation with 4\% paraformaldehyde. Cells were then stained with Apollo Dye Solution and mounted with Hoechst 33342. EdU positive cells were photographed and counted under light microscopy.

\section{Statistical analysis}

Statistical analysis was performed using SPSS version 19.0 software. All numerical data are presented as mean \pm standard deviation (SD). Parametric variables were compared using $t$-tests. Survival was assessed according to the Kaplan-Meier method and compared using the log-rank test. Differences were considered statistically significant when $\mathrm{P}<0.05$.

\section{Results}

\section{TNFRSF6B mRNA expression}

The expression of TNFRSF $6 B$ mRNA was analyzed using the TCGA database. TNFRSF6B expression was significantly elevated in several solid tumors including pancreatic adenocarcinoma (PAAD), colon adenocarcinoma (COAD), head and neck squamous cell carcinoma (HNSC), and stomach adenocarcinoma (STAD) (Figure 1A). The ONCOMINE database analysis of 19 datasets revealed that TNFRSF6B mRNA expression was increased in colorectal cancer, gastric cancer, kidney cancer, and pancreatic cancer compared to normal tissues (Figure 1B). Metaanalysis of TNFRSF6B expression in the ONCOMINE database revealed that the median rank was 704 and the $\mathrm{P}$ value was $1.97 \times 10^{-7}$ (Figure 1C). Subsequent experiments 
A

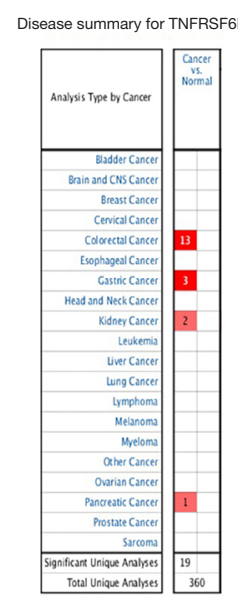

B

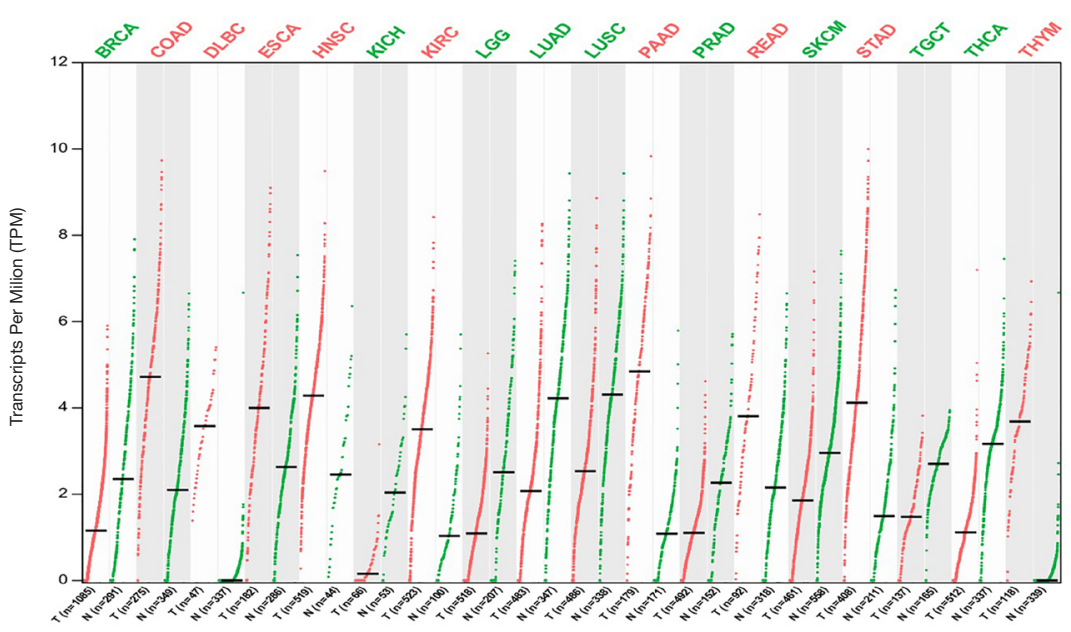

C

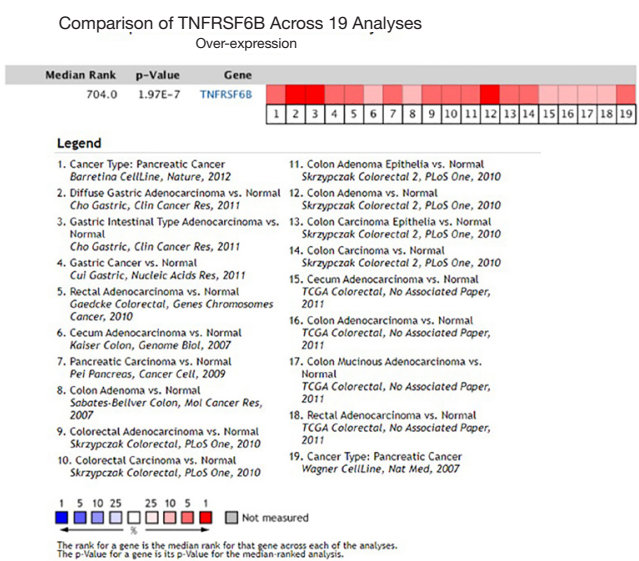

$\mathrm{D}$

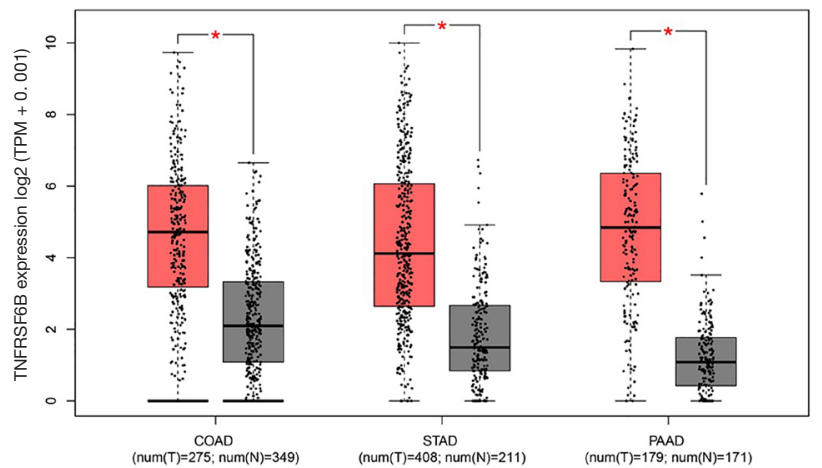

$\mathrm{E}$
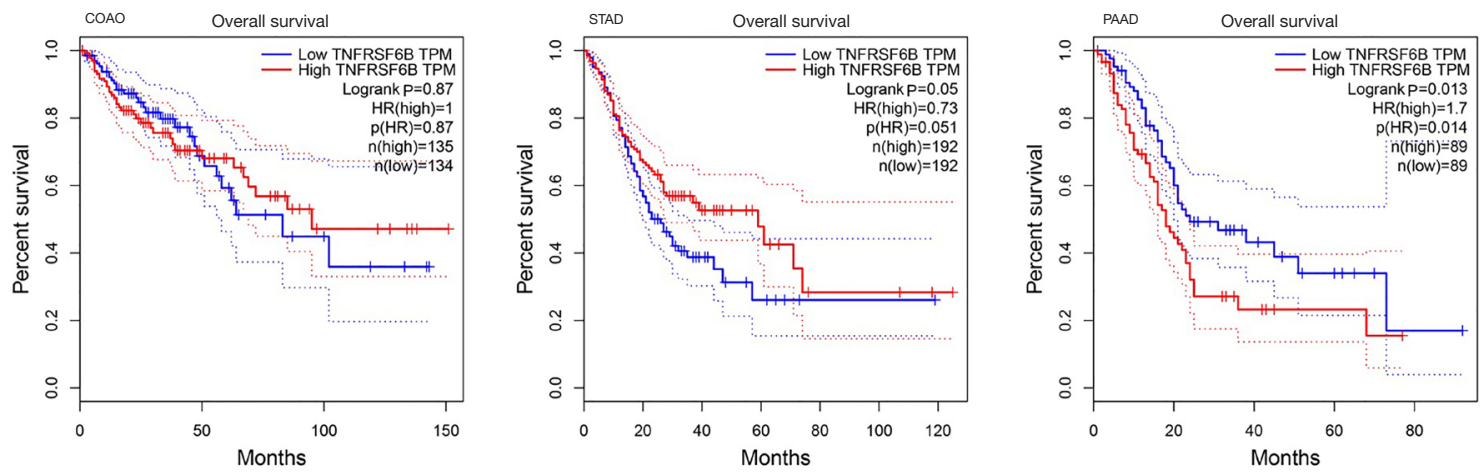

Figure 1 TNFRSF6B expression levels in human cancers. (A) The expression of TNFRSF6B in different tumor types from the TCGA database were determined by GEPIA (P value cutoff: 0.0001; red represents upregulated expression and green represents downregulated expression). (B) TNFRSF6B expression in data sets of different cancers in the ONCOMINE database. This graph generated by ONCOMINE indicates the number of datasets with statistically significant mRNA overexpression (red) of TNFRSF6B (cancer tissues vs. corresponding normal tissues). The threshold was defined with the following parameters: $\mathrm{P}$ value of 1E-4, fold change of 2, and gene ranking of 10\%. (C) Meta-analysis of TNFRSF6B mRNA expression in 19 analyses in the ONCOMINE database. (D) The GEPIA database verified that TNFRSF6B expression was significantly upregulated in COAD, $\mathrm{STAD}$, and PAAD compared with normal tissues. *, $\mathrm{P}<0.05$. (E) The relationship between levels of TNFRSF6B expression and prognosis of CAOD, STAD. and PAAD. TNFRSF6B, tumor necrosis factor receptor superfamily member 6b; TCGA, The Cancer Genome Atlas; GEPIA, Gene Expression Profiling Interactive Analysis; COAD, colon adenocarcinoma; STAD, stomach adenocarcinoma; PAAD, pancreatic adenocarcinoma. 
focused on gastrointestinal tumors. The GEPIA database further confirmed that TNFRSF $6 B$ was upregulated in PAAD, COAD, and STAD compared to normal tissues (Figure 1D). Kaplan-Meier plots showed that increased expression of TNFRSF $6 B$ mRNA was significantly associated with $\mathrm{OS}$ in PAAD patients $(\mathrm{P}=0.013)$, but not in COAD patients $(\mathrm{P}=0.87)$, nor in STAD patients $(\mathrm{P}=0.05)$ (Figure 1E). Therefore, the involvement of TNFRSF6B in the network regulation of pancreatic cancer was further investigated.

\section{The interaction network analysis of TNFRSF6B}

To further study the biological function of TNFRSF6B in pancreatic cancer and the role of the regulatory network, genes related to TNFRSF6B were analyzed. The heat map shows the top 50 important genes that were positively or negatively correlated with TNFRSF6B (Figure 2A). GO and KEGG analyses of these genes were carried out to explore the potential functional pathways related to TNFRSF $6 B$. The results demonstrated that TNFRSF6B-related genes were mainly involved in cytokine-mediated signaling pathways, myeloid leukocyte activation, ECM organization, response to type I interferon, cellular response to tumor necrosis factor, apoptotic signaling pathways, and actin filament-based processes (Figure 2B). The Clusterprofiler package was used for GSEA analysis of TNFRSF6B-related genes, including GO, KEGG, and Reacteame analyses. The GO results revealed that TNFRSF6B was mainly involved in immune effector processes, immune responses, regulation of immune system processes, signaling receptor binding, cell adhesion, positive regulation of signal transduction, cell proliferation, regulation of cell death, apoptotic processes, and cell cycle processes. (Figure 2C). The KEGG results showed that TNFRSF6B was enriched in the cell cycle, the p53 signaling pathway, ECM-receptor interaction, the NFkappa B signaling pathway, apoptosis, and the NOD-like receptor signaling pathway (Figure $2 D$ ). The Reacteame results demonstrated that TNFRSF6B was widely involved in ECM organization, cell cycle checkpoints, degradation of the ECM, cell cycle, apoptosis, cytokine signaling in the immune system, and signaling by the interleukin innate immune system. (Figure 2E). These results suggested that TNFRSF6B is involved in the cell cycle, apoptosis, tumorrelated signal transduction, and immune regulation in pancreatic cancer.

\section{Construction of the weighted gene co-expression network analysis (WGCNA) and analysis of bub genes}

The optimal soft threshold of mRNA co-expression network analysis was 11 (Figure $3 A$ ), and the systematic clustering tree of genes was obtained by using the WGCNA according to the heterogeneity between genes (Figure 3B). According to the dynamic mixed shearing method, the core gene cluster was identified and five gene modules were obtained (Figure 3C). Among these modules, the MEbrown module was found to be closely related to OS time ( $r=-0.25$, $\mathrm{P}=0.001)$, DSS time $(\mathrm{r}=-0.25, \mathrm{P}=0.001)$, PFI time $(\mathrm{r}=-0.29$, $\mathrm{P}=2 \mathrm{e}-04)$, and DFI ( $\mathrm{r}=-0.33, \mathrm{P}=2 \mathrm{e}-05)$.

A total of 30 genes were extracted from the MEbrown module, and 10 of the most meaningful hub genes were screened by cytoHubba, namely, $S 100$ calcium binding protein A11(S100A11), gap junction protein beta 3 (G7B3), tumor associated calcium signal transducer 2 (TACSTD2), keratin 7 (KRT7), potassium calcium-activated channel subfamily $N$ member 4 (KCNN4), capping actin protein, gelsolin like $(C A P G)$, integrin subunit beta 4 (ITGB4), laminin subunit beta 3 (LAMB3), stratifin (SFN), and keratin 19 (KRT19). GeneMANIA was used to construct the PPI network of TNFRSF6B and the hub genes (Figure 3D). CLUEGO was used for biological function annotation analysis. The results demonstrated that these 10 hub genes were widely involved in ECM-receptor interaction, cell adhesion, cell cycle, and other processes (Figure 3E).

A heat map of the 10 hub genes was constructed under conditions of high and low TNFRSF6B expression (Figure S1A). GEPIA analysis showed that the expression of these 10 key genes in pancreatic cancer was higher than that in normal tissues (Figure S1B). With the exception of ITGB4 $(\mathrm{P}=0.078)$ and TACSTD2 $(\mathrm{P}=0.087)$, the other eight genes showed significant correlation with prognosis (Figure S1C), and thus, may function as potential prognostic markers for pancreatic cancer.

\section{TNFRSF6B and immune cell infiltration}

Above network analysis revealed that TNFRSF6B is highly related to the immune response. According to the median value of TNFRSF6B, immune cells were divided into the high expression group and the low expression group. A heat map of 29 types of immune cells was constructed (Figure 4A), and the correlation between TNFRSF6B and these immune cells was analyzed (Figure $4 B$ ). The results 
A

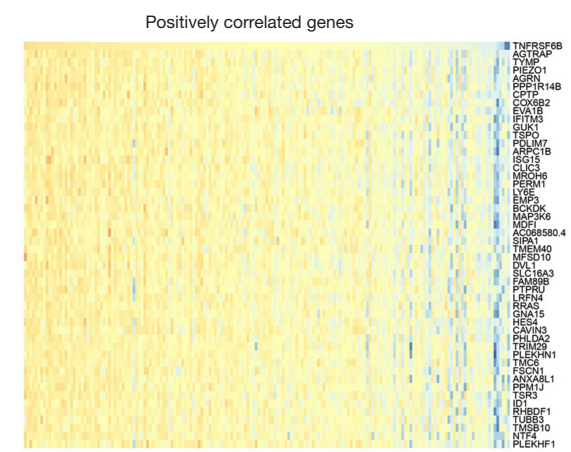

B
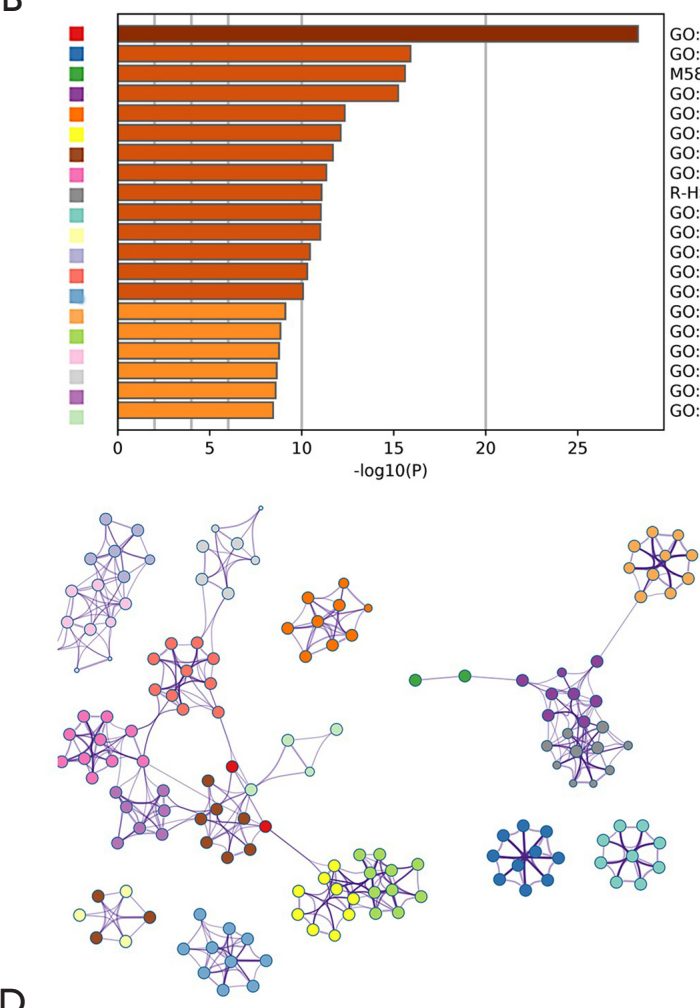

D

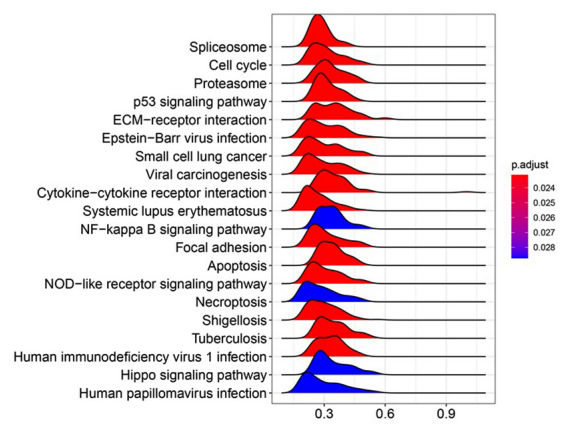

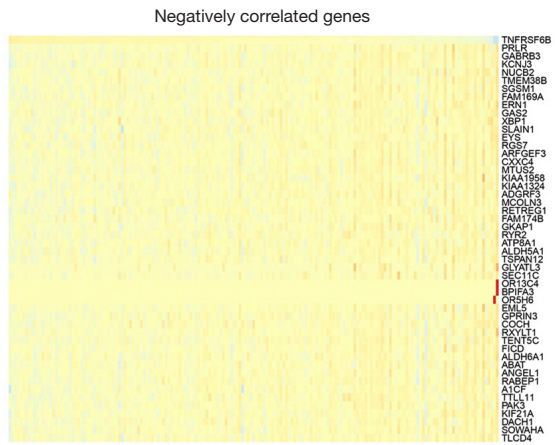

GO:0019221: cytokine-mediated signaling pathway GO:0002274: myeloid leukocyte activation M5885: NABA MATRISOME ASSOCIATED

GO:0030198: extracellular matrix organization GO:0009611: response to wounding O:0034340: response to type I interferon O:0071356: cellular response to tumor necrosis factor GO:1903530: regulation of secretion by cell R-HSA-1474290: Collagen formation R:0070268: cornification

:0048729: tissue morphogenesis

O:0097190: apoptotic signaling pathway

G0:0030029: actin filament-based pron

GO:0022411: cellular component disassems

GO:0043903: regulation of symbiosis, encompassing mutualism through parasitism

O.0008630: intrinsic apoptotic signaling pathway in response to DNA damage

GO:0031589: cell-substrate adhesion

GO:0032496: response to lipopolysaccharide

C

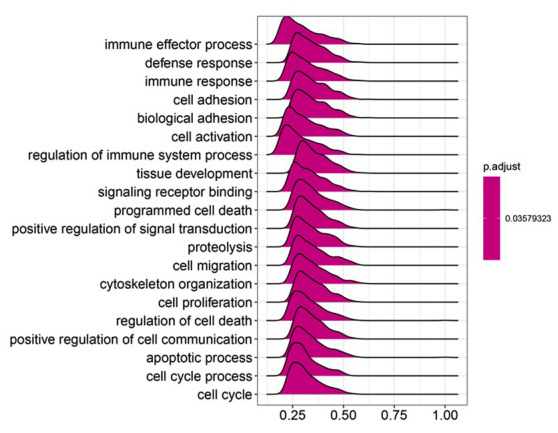

$E$

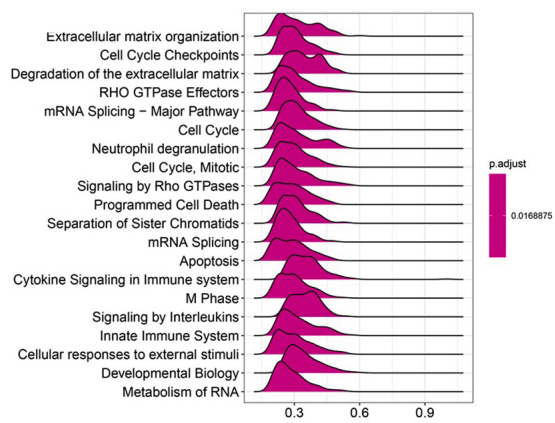

Figure 2 Interaction network analysis of TNFRSF6B. (A) Heat maps showing the top 50 genes that were positively and negatively correlated with TNFRSF6B in PAAD samples. (B) A heatmap of Gene Ontology analysis including BP, CC, and MF. (C) Gene Ontology analysis, (D) KEGG pathway analysis, and (E) Reacteame analysis of genes correlated with TNFRSF6B using Clusterprofiler. TNFRSF6B, tumor necrosis factor receptor superfamily member 6b; PAAD, pancreatic adenocarcinoma; BP, biological process; CC, cellular component; MF, molecular function; KEGG, Kyoto Encyclopedia of Genes and Genomes. 
A
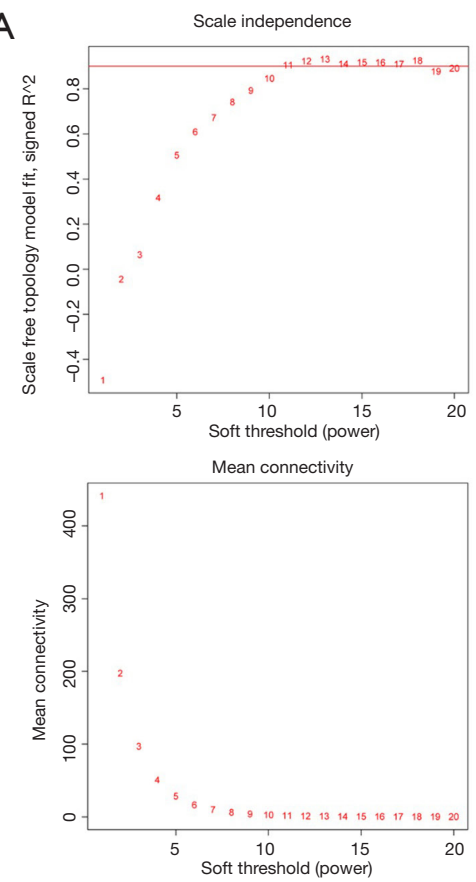

D

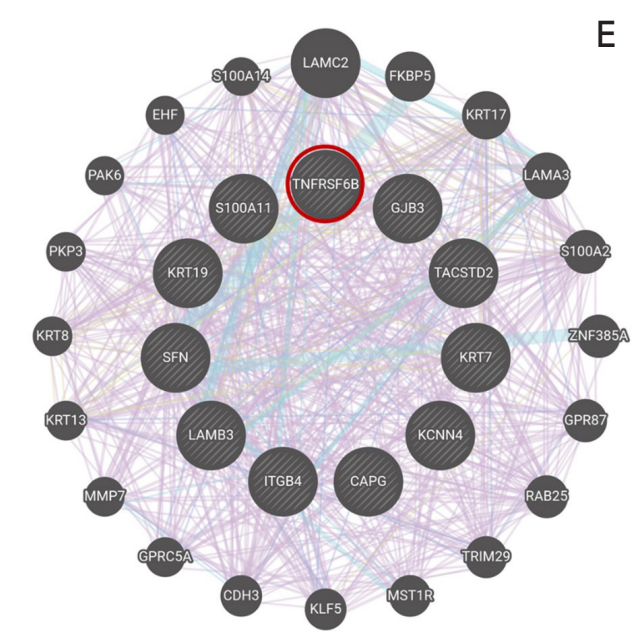

B

C
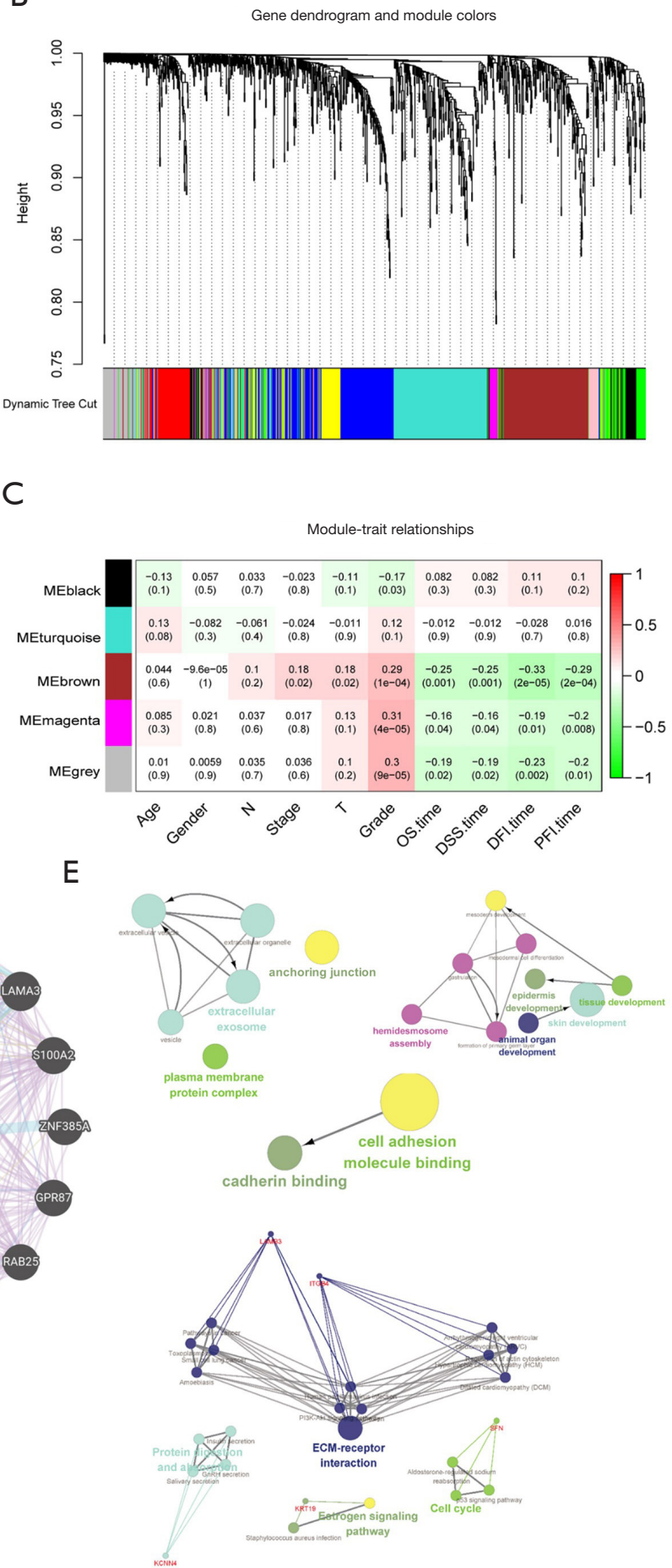

Figure 3 Weighted gene co-expression network analysis of gene expression data. (A) Analysis of scale-free fit index and the mean connectivity for various soft-thresholding powers. (B) Hierarchical clustering dendrogram of genes with dissimilarity based on topological overlap. Modules are the branches of the clustering tree. (C) Correlation between module eigengenes and clinical traits. Each row corresponds to a module eigengene and columns represent clinical traits. Each cell contains the correlation and $\mathrm{P}$ value. (D) Protein-protein interaction network of TNFRSF6B and hub genes by GeneMANIA. (E) The biological process analysis of hub genes was performed using the ClueGO plug-in. $\mathrm{P}<0.05$ was considered to be statistically significant. TNFRSF6B, tumor necrosis factor receptor superfamily member $6 \mathrm{~b}$. 
A

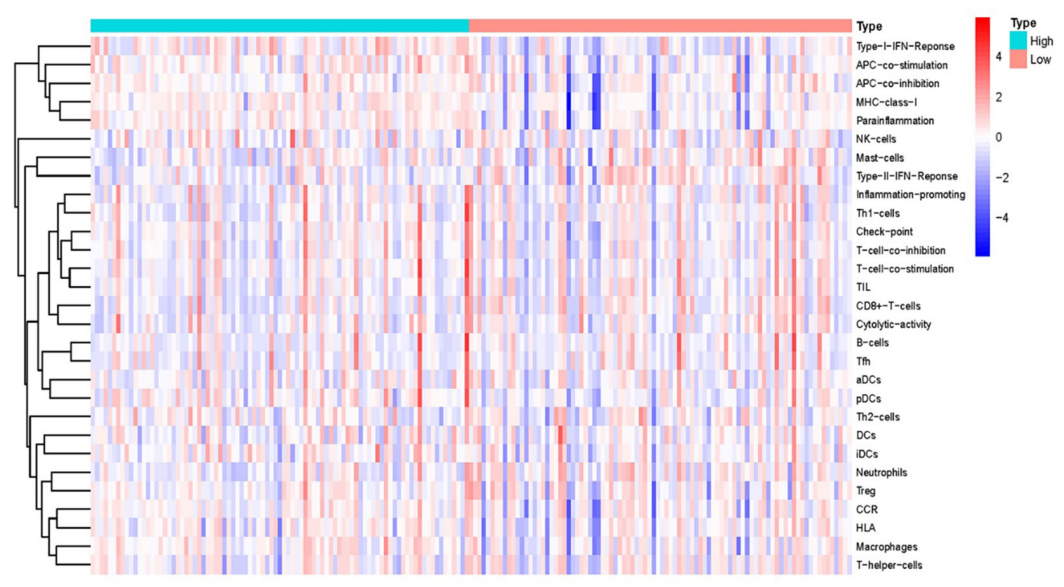

B

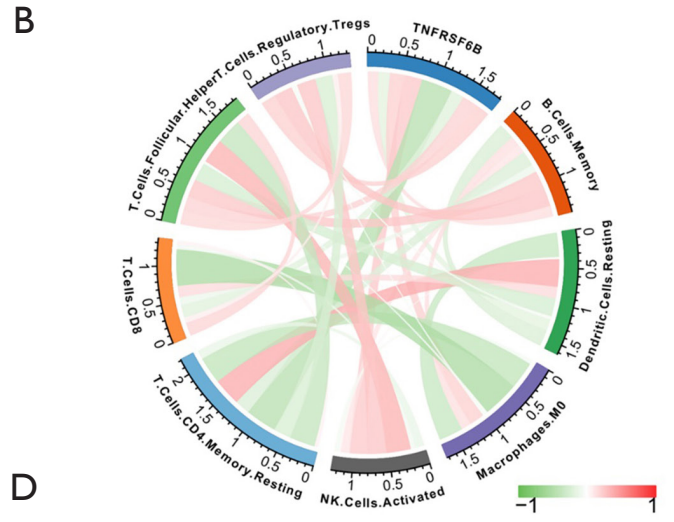

C
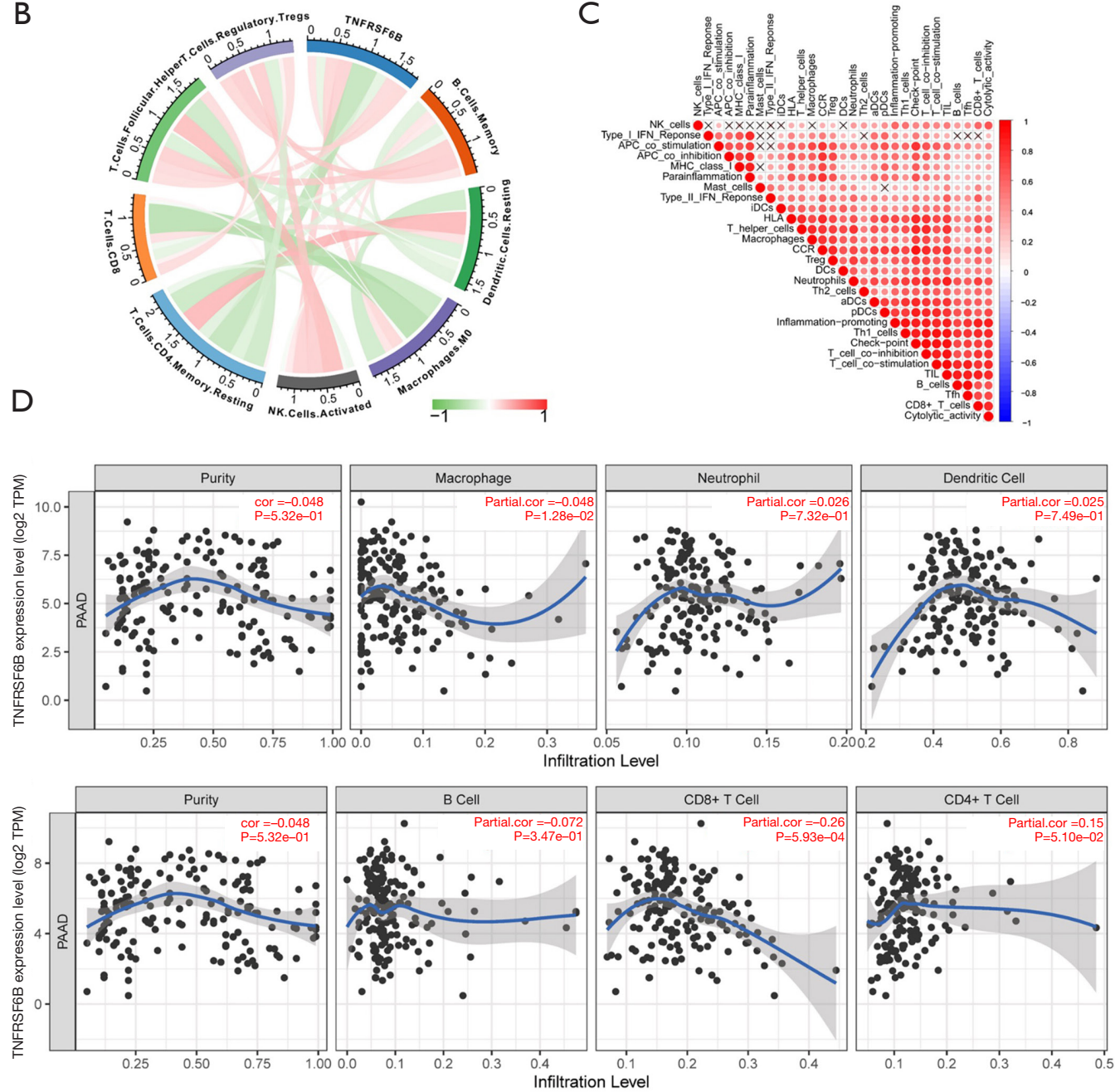

Figure 4 The relationship between TNFRSF6B expression and immune cell infiltration. (A) The correlation between TNFRSF6B expression and immune cells. Red color represents positive correlation and blue color represents negative correlation. (B) The correlation between TNFRSF6B and 29 immune cell types. The color and the values indicate the Spearman correlation coefficient. (C) TNFRSF6B expression is correlated with immune cells in the TCGA database. (D) Correlation between TNFRSF6B expression and immune cells in the TIMER database. TNFRSF6B, tumor necrosis factor receptor superfamily member 6b; TCGA, The Cancer Genome Atlas; TIMER, Tumor Immune Estimation Resource. 
revealed that TNFRSF6B was related to Type-II interferonresponse, CD8+ T cells, mast cells, and neutrophils (Figure S2). Circle mapping was performed on immune cell types with a Pearson's correlation coefficient $r>0.15$. TNFRSF6B was negatively correlated with CD8+ T cells $(\mathrm{r}=-0.1814, \mathrm{P}=0.025), \mathrm{CD} 4+$ memory $\mathrm{T}$ cells $(\mathrm{r}=-0.4430$, $\mathrm{P}<0.001)$, and macrophages ( $\mathrm{M} 0 ; \mathrm{r}=-0.2102, \mathrm{P}=0.009)$, and positively correlated with regulatory $\mathrm{T}$ cells (Treg; $\mathrm{r}=0.2136$, $\mathrm{P}=0.008$ ) (Figure $4 C$ ). Analysis of the TIMER database demonstrated that TNFRSF $6 B$ was negatively correlated with $\mathrm{CD} 8+\mathrm{T}$ cells $(\mathrm{r}=-0.4, \mathrm{P}<0.001)$ and macrophages $(\mathrm{r}=-0.19, \mathrm{P}=0.013)$ (Figure $4 D$, Figure S3). The relationship between TNFRSF $6 B$ and immune checkpoints was further examined and shown in heatmap (Figure $5 A$ ). The expression of Fas ligand (FASLG), CD40 ligand (CD40LG), inducible T Cell costimulator ligand (ICOSLG), and CD28 decreased significantly in the TNFRSF6B high expression group compared to the low expression group $(\mathrm{P}=0.015$, $\mathrm{P}=0.002, \mathrm{P}=0.018$, and $\mathrm{P}=0.018$, respectively) (Figure $5 B$ ).

\section{The effects of TNFRSF6B on cell proliferation and invasion in vitro}

To verify the results of the bioinformatics analysis, shRNA was used to knock out TNFRSF6B gene expression in the PANC-1 pancreatic cancer cell line. EdU assays revealed that cell proliferation was significantly impaired in PANC-1 cells transfected with sh-TNFRSF6B (sh-TNFRSF6B cells) compared with cells transfected with the negative control shRNA (sh-NC cells) $(\mathrm{P}<0.01$, Figure $6 A)$. A similar result was observed in the colony formation assays $(\mathrm{P}<0.0001$, Figure $6 B)$. Transwell migration assays demonstrated that knockout of the TNFRSF $6 B$ gene significantly reduced the invasion ability of pancreatic cancer cells $(\mathrm{P}<0.01$, Figure $6 C$ ). These results suggested that knockout of the TNFRSF $6 B$ gene inhibited the proliferation and clone formation ability of pancreatic cancer cells.

\section{Interaction network analysis of TNFRSF6B targeted genes}

The gene chip data of pancreatic cancer samples from the TNFRSF $6 B$ down-regulation cells and the negative control cells were analyzed. A heat map of the expression levels was constructed, with red representing high expression and green representing low expression (Figure $7 A$ ). The downstream genes of TNFRSF $6 B$ were identified and the network regulation in sh-TNFRSF $6 B$ and sh-NC cells were examined. The GO results showed that these genes were enriched in the Type 1 interferon signaling pathway, cellular response to type 1 interferon, response to type 1 interferon, and the cyclin-dependent protein kinase holoenzyme complex (Figure $7 B$ ). The KEGG analysis revealed that the important pathways included the JAKSTAT signaling pathway, the NOD-like receptor signaling pathway, necroptosis, the prolactin signaling pathway, the Toll-like receptor signaling pathway, the C-type lectin receptor signaling pathway, the TNF signaling pathway, the retinoic acid-inducible gene I (RIG-I)-like receptor signaling pathway, the p53 signaling pathway, and the NFkappa B signaling pathway (Figure $7 C$ ). Based on the IPA, the regulatory effects of TNFRSF $6 B$ were analyzed and the possible pathways of the upstream regulatory networks and the downstream functions involving the differential genes were identified. The results revealed that upstream regulators including interferon (INF)- $\alpha$, INF- $\beta$, INF- $\gamma$, and STAT1 were involved in the regulation of glomerular cell apoptosis, cryostasis of tumor cell lines, and the antiviral response of cells through cyclin D1 (CCND1), cyclin dependent kinase inbibitor $1 A(C D K N 1 A)$, carcinoembryonic antigen-related cell adhesion molecule 1 (CEACAM1), interferon regulatory factor 1 (IRF1) and other genes (Figure $7 D$ ).

The relationship between TNFRSF6B and CEACAM1 was analyzed using UCSC Xena. Consistent correlation patterns were observed between these two genes in different patients (Figure 8A). This positive correlation was substantiated by analysis via the TIMER2.0 database (Figure $8 B$ ). Western blot assays confirmed that CEACAM1 was regulated by TNFRSF $6 B$, and the expression of the $C E A C A M 1$ protein was down-regulated after knockout of the TNFRSF6B gene (Figure 8C). TNFRSF6B and CEACAM1 were found to be enriched in circadian entrainment, gap junction, tyrosine metabolism, phenylalanine metabolism, and other pathways (Figure $8 D$ ). Immunofluorescence analysis showed that TNFRSF $6 B$ and CEACAM1 were mainly expressed in the cytoplasm (Figure $8 E$ ). The interactions between TNFRSF6B and CEACAM1 were also confirmed with immune co-precipitation assays (Figure $8 F$ ).

\section{Discussion}

Pancreatic cancer remains highly malignant with poor patient prognosis, which is largely due to high invasiveness, late diagnosis and low resection rates (2). Furthermore, pancreatic cancer is insensitive to radiotherapy and chemotherapy $(25,26)$. Compared to conventional therapies, gene therapy is an emerging and dynamic area 
A

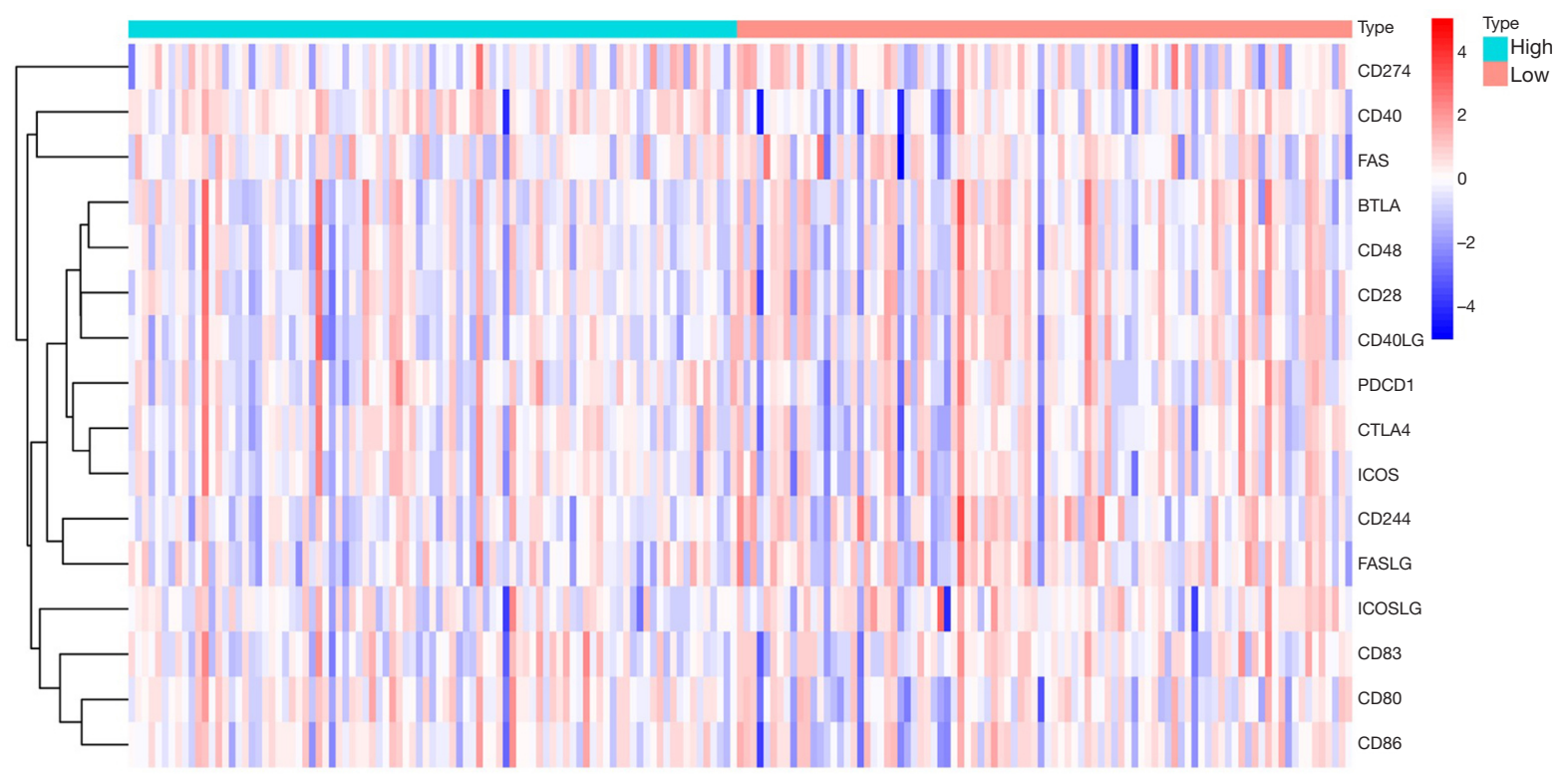

B
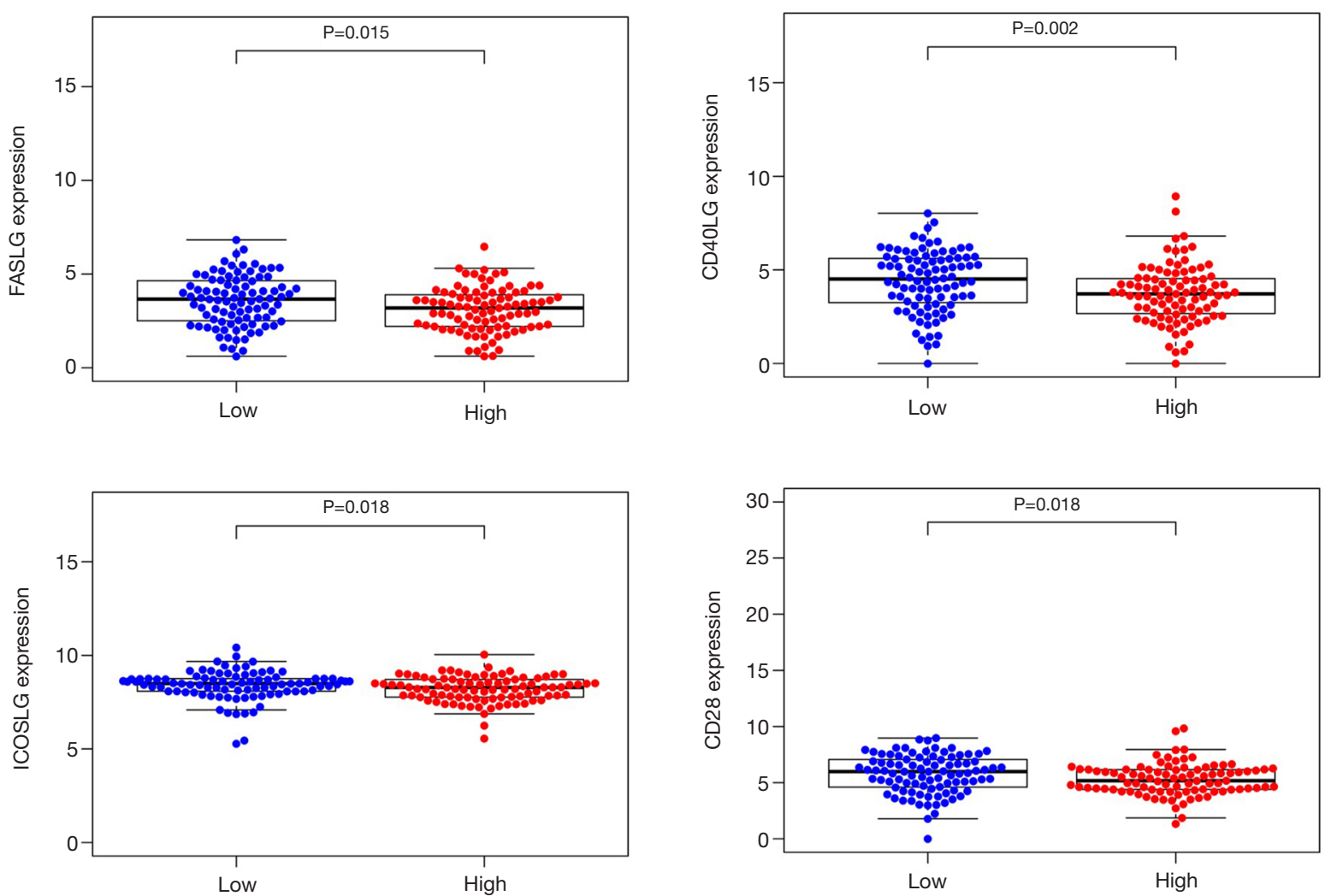

Figure 5 The correlation between TNFRSF6B expression and immune checkpoints. (A) A heatmap representation of the correlation between TNFRSF6B expression and checkpoint-associated genes. Red color represents positive correlation and blue color represents negative correlation. (B) The correlation between TNFRSF6B expression and the four immune checkpoints FASLG, CD40LG, ICOSLG, and CD28. TNFRSF6B, tumor necrosis factor receptor superfamily member 6b; FASLG, Fas ligand; CD40LG, CD40 ligand; ICOSLG, inducible $T$ cell costimulator ligand. 
A
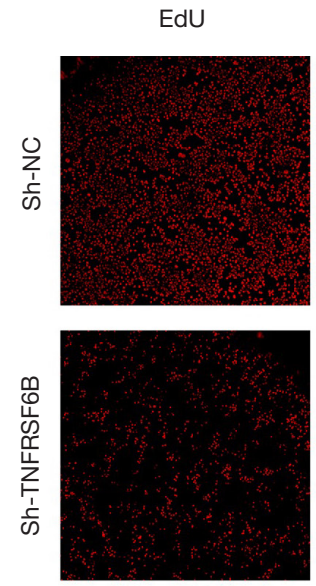

B

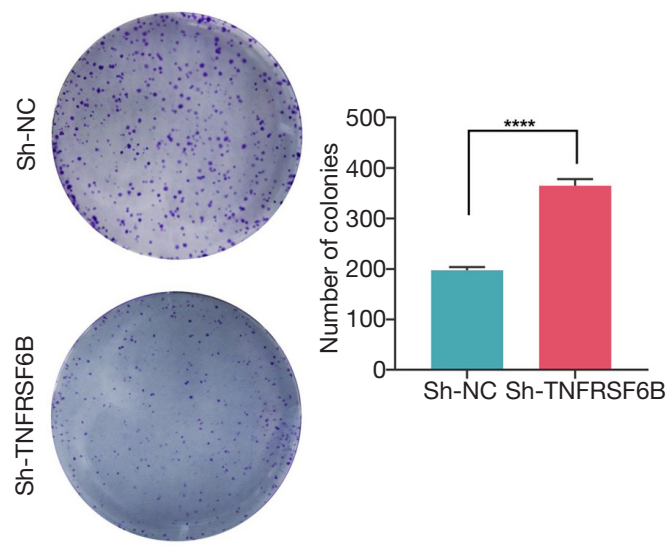

Hoechst
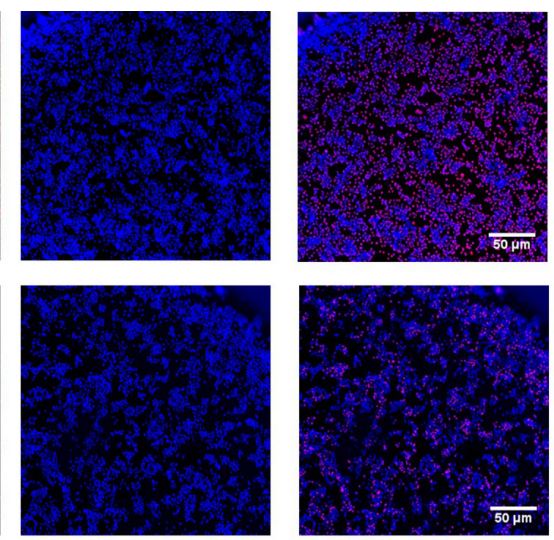

C

Merge
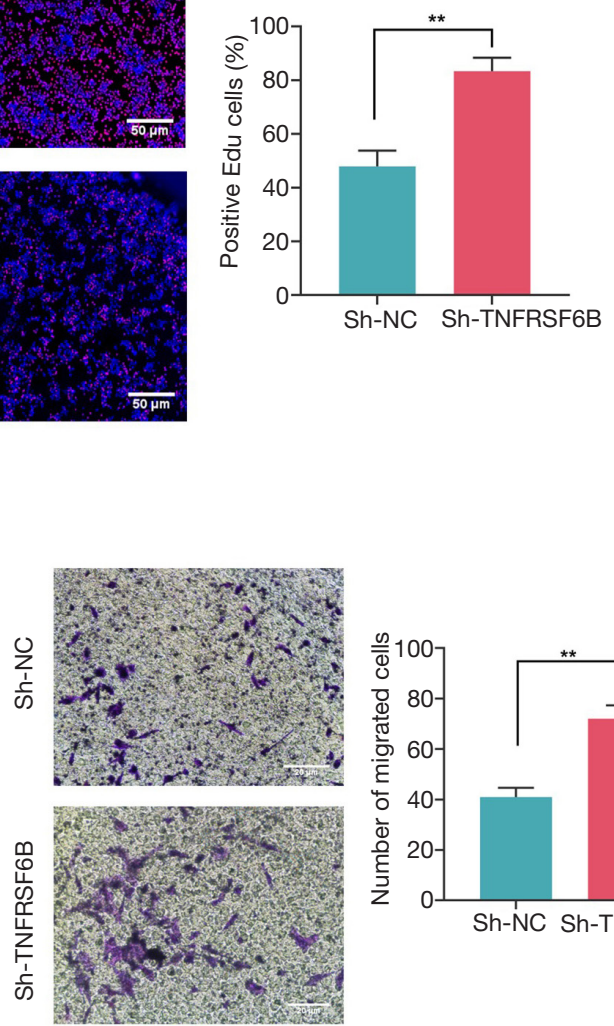

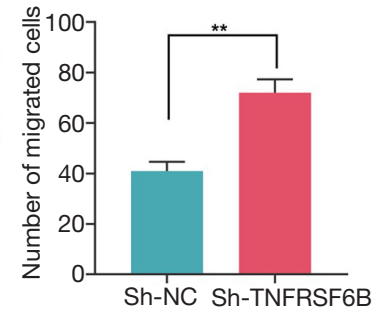

Figure 6 TNFRSF6B exhibits oncogenic roles in pancreatic cancer cells. (A) Cell proliferation was evaluated using EdU incorporation assays. **, $\mathrm{P}<0.01$. (B) Colony formation assays were performed on pancreatic cancer cells transfected with sh-TNFRSF6B or the negative control (sh-NC). ***, $\mathrm{P}<0.0001$. (C) The migration and invasion ability of TNFRSF6B cells were evaluated by Transwell assays. **, $\mathrm{P}<0.01$. TNFRSF6B, tumor necrosis factor receptor superfamily member 6b; sh, short hairpin; NC, negative control.

of research, in which enhanced immune surveillance of tumor cells has attracted considerable attention. According to American Society of Clinical Oncology (ASCO) guideline, Poly ADP-ribose Polymerase (PRAP) inhibitor olaparib is recommended for treatment of patients with pancreatic cancer who have a germline BRCA1 or BRCA2 mutation. Besides, in patients with tumors harboring neurotrophic tyrosine kinase (NTRK) fusions, treatment with larotrectinib or entrectinib is recommended. Although olaparib, larotrectinib and entrectinib are treatment options after first-line therapy, more clinical trials are needed for confirmation of therapeutic effect of these drugs (27).

In this study, the expression levels of TNFRSF6B in PC was first analyzed using the TCGA and ONCOMINE databases. TNFRSF6B was found to be upregulated in PAAD samples compared to normal tissues, and TNFRSF6B expression was correlated with OS of patients with pancreatic cancer. Enrichment analysis of genes related to TNFRSF6B revealed important networks, including the tumor necrosis factor signaling pathway, the p53 signaling pathway, the interferon response pathway, and the apoptosis signaling pathway. In addition, TNFRSF6B was found to be involved in the regulation of immune responses, immune process regulation, cell proliferation, cell cycle, and apoptosis. To confirm the accuracy of the cancer database, the expression of TNFRSF6B was silenced in PANC-1 using shRNA, and the results were consistent with the prediction of the TCGA and ONCOMINE databases. Furthermore, 
A
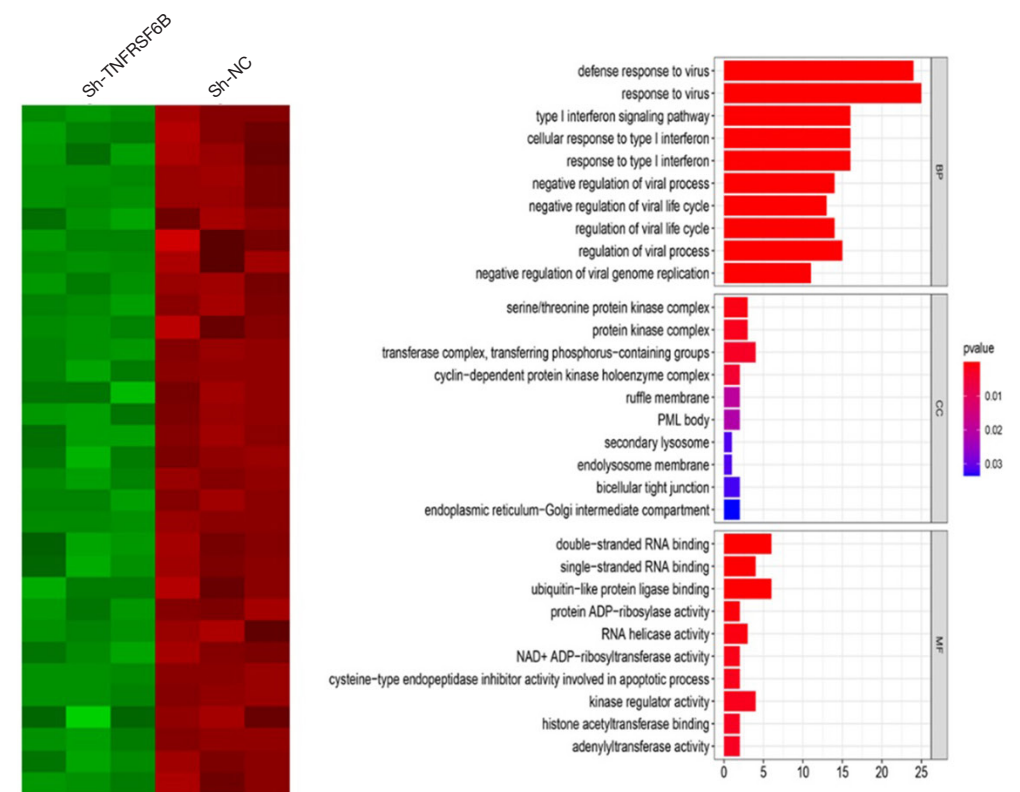

C

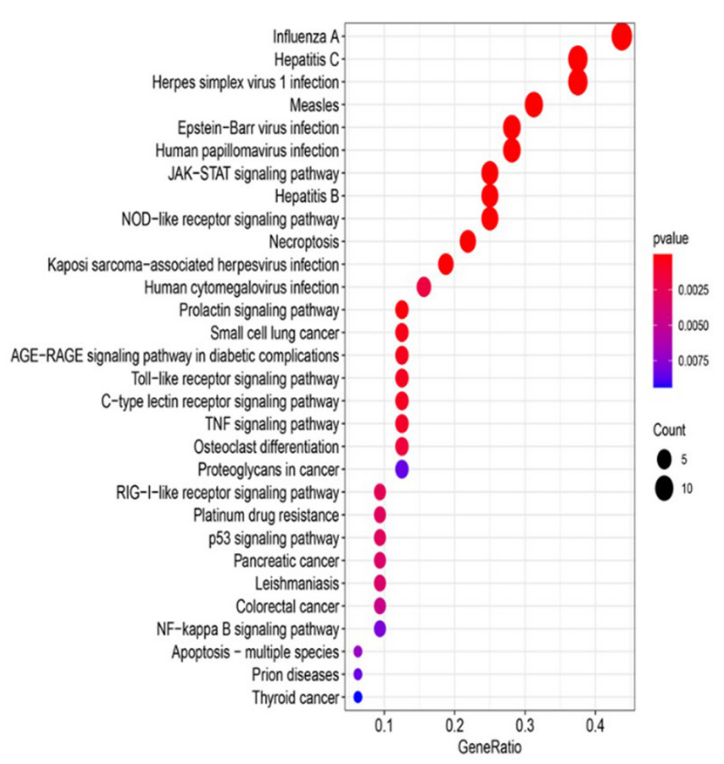

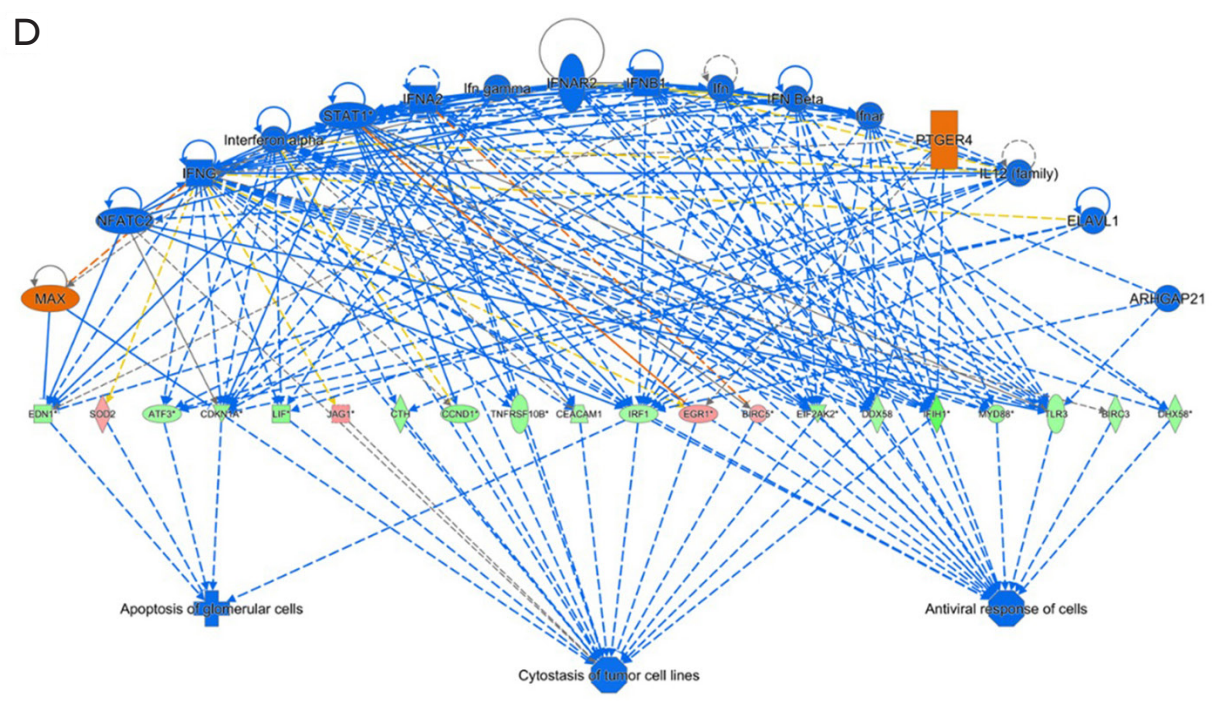

Figure 7 Analysis of the pathways and biological interaction networks of genes targeted by TNFRSF6B. (A) A heatmap representation of the differential genes in sh-TNFRSF6B cells and sh-NC cells. (B) Gene Ontology (GO) analysis indicated that genes regulated by TNFRSF6B were involved in biological processes (BP), cellular components $(\mathrm{CC})$, and molecular function $(\mathrm{MF})$. $\mathrm{P}<0.05$. (C) A bar chart showing the KEGG pathway analysis. (D) Analysis of the regulatory effect based on IPA. TNFRSF6B, tumor necrosis factor receptor superfamily member 6b; sh, short hairpin; NC, negative control; KEGG, Kyoto Encyclopedia of Genes and Genomes; IPA, ingenuity pathway analysis.

this study revealed that TNFRSF $B$ can promote proliferation, colony formation capacity, and invasion of pancreatic cancer cells.

The TNFRSF6B gene (1,114 bp) is located on human chromosome 20q13.3. It encodes the soluble protein DcR3 which consists of 271 amino acid residues, including four cysteine residue rich regions (CRDs) and an $\mathrm{N}$-glycosylation site at the asparagine residue at position 173. TNFRSF6B is overexpressed in a variety of tumor tissues and cells, including esophageal cancer (28), gastric cancer (14), 
A

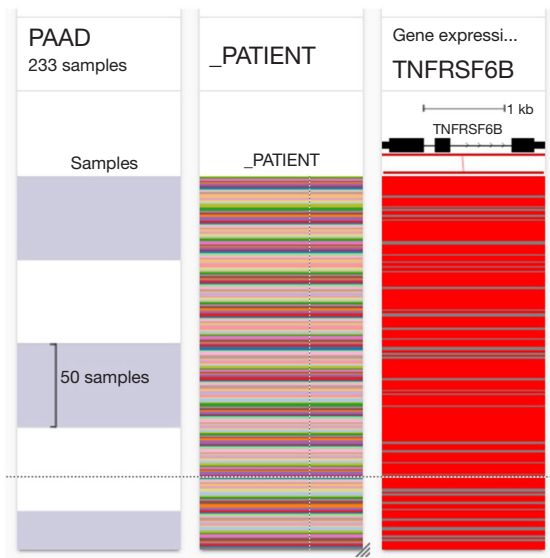

C

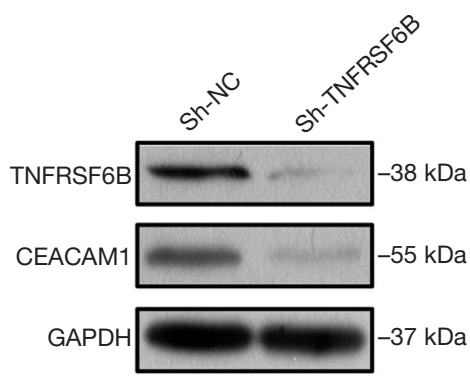

E

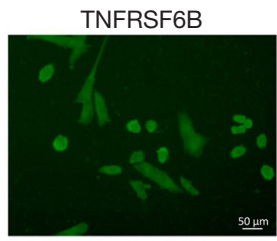

DAPI
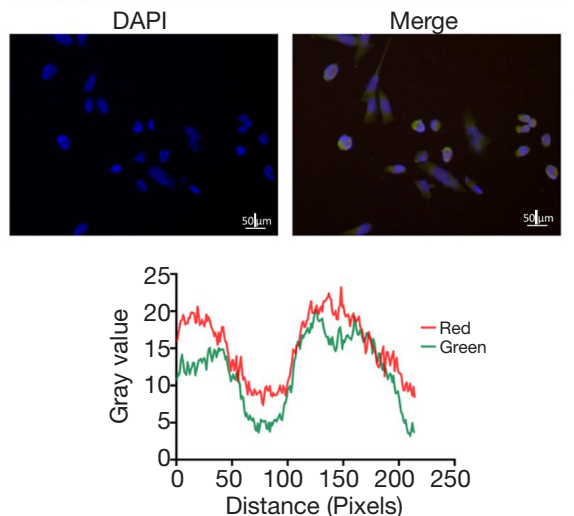

B

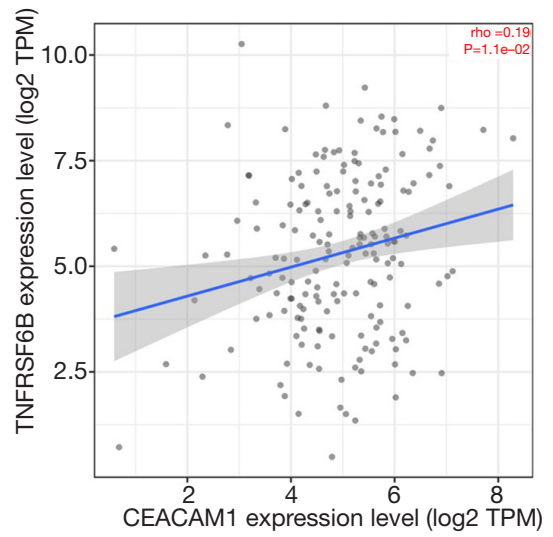

D

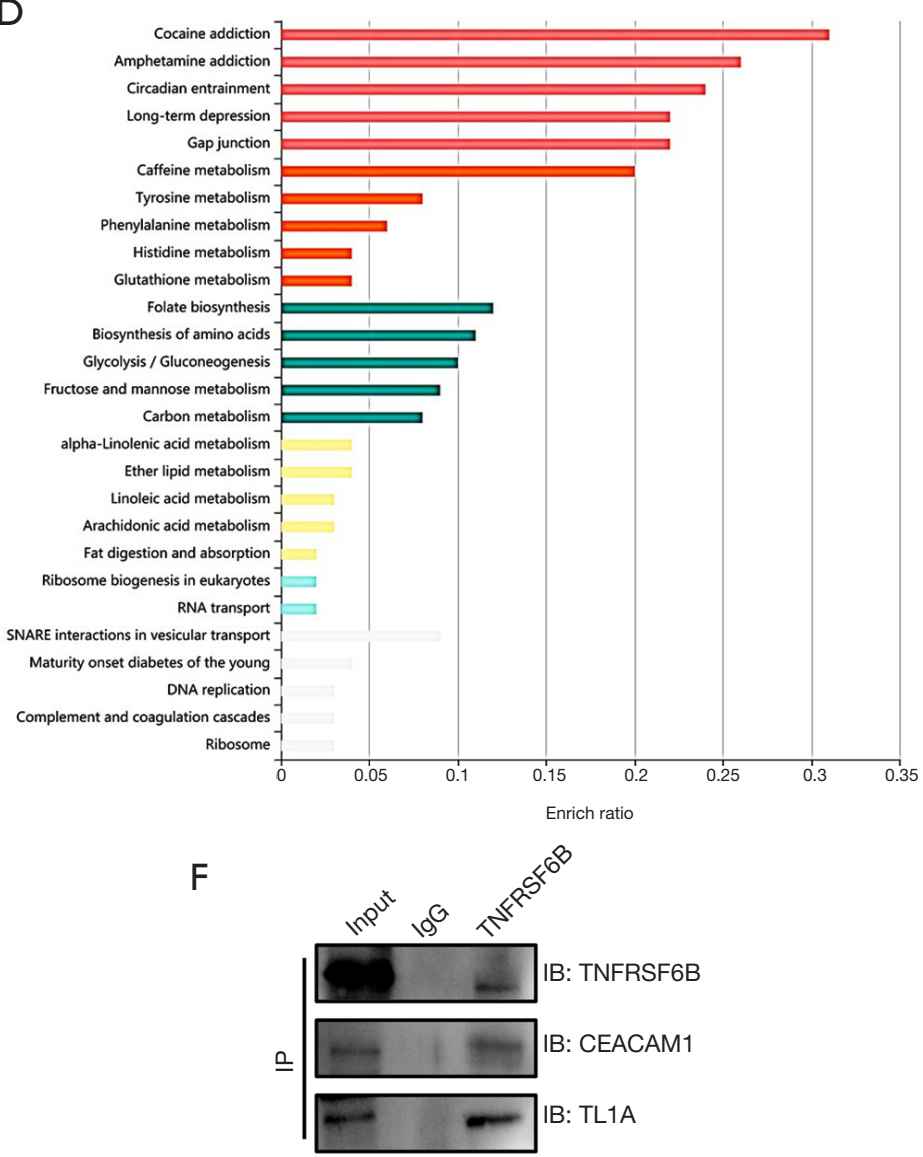

Figure 8 Expression of TNFRSF6B and CEACAM1 were highly correlated. (A) A heatmap of TNFRSF6B and CEACAM1expression across PAAD samples in the TCGA database using UCSC Xena. (B) The correlation between TNFRSF6B and CEACAM1 expression was assessed using the TIMER database. (C) TNFRSF6B and CEACAM1 protein expression in sh-NC and sh-TNFRSF6B cells was determined by Western blotting. (D) Interacting proteins of TNFRSF6B and CEACAM1 were analyzed using KOBAS 3.0. (E) Immunofluorescence staining of TNFRSF6B and CEACAM1 in PANC-1 cells. The scale bars represent $50 \mu \mathrm{m}$. (F) Co-immunoprecipitation (CoIP) was used to identify the interactive proteins. TL1A was used as the positive internal reference. TNFRSF6B, tumor necrosis factor receptor superfamily member 6b; CEACAM1, carcinoembryonic antigen-related cell adhesion molecule 1; PAAD, pancreatic adenocarcinoma; TCGA, The Cancer Genome Atlas; TIMER, Tumor Immune Estimation Resource; sh, short hairpin; NC, negative control; KOBAS, Kyoto Encyclopedia of Genes and Genomes (KEGG) Orthology-Based Annotation System; TL1A, tumor necrosis factor-like cytokine 1A. 
colon cancer $(17,18)$, pancreatic cancer $(15,16)$, lung cancer $(12,13)$, glioblastoma multiforme (29), renal cell carcinoma (30), ovarian cancer (31), virus-associated lymphoma (32), diffuse B-cell lymphoma (33), multiple myeloma (34), oral malignancies (35) and hepatocellular carcinoma (36). Overexpression of TNFRSF $6 B$ can inhibit apoptosis and promote immune escape of tumor cells. TNFRSF $6 B$ can act both as a "decoy" and a "non-decoy". As a decoy, TNFRSF6B competitively binds with FasL, TL1A, and LIGHT that are expressed on T cells (13), blocking their ability to induce apoptosis and clear tumor cells, thereby contributing to the pathogenesis of tumors (14). In its "non-decoy" function, TNFRSF6B plays an important role in cellular regulation (37) by affecting the activity and differentiation of $\mathrm{T}$ lymphocytes, macrophages, and dendritic cells (DC). It can also downregulate the immune system and facilitate tumor angiogenesis (38-41).

Indeed, analysis of the TCGA and ONCOMINE databases revealed that TNFRSF $6 B$ was related to immune cells such as CD8+ T cells, mast cells, neutrophils, and macrophages. There are several possible mechanisms by which TNFRSF6B can downregulate T lymphocytes. First, TNFRSF6B can inhibit the polymerization of actin in $\mathrm{T}$ cells stimulated by mitogen, inhibit the formation of pseudopodia, and inhibit the interaction between $\mathrm{T}$ cells and antigen presenting cells (42). Second, LIGHT provides T lymphocytes with CD28-independent costimulatory signals and induces the secretion of T helper type 1 (Th1) cytokines such as IFN- $\gamma$ to participate in the tumor immune response. TL1A induces the activation of NF-kappa B, which acts as a co-stimulating factor for the initiation of T lymphocytes. TNFRSF6B can bind competitively with both LIGHT and TL1A to block the activation of T cells by LIGHT and TL1A (43). Third, TNFRSF6B can downregulate the costimulatory molecules of $\mathrm{T}$ lymphocytes such as CD80, CD40, and CD54 (44). Furthermore, TNFRSF6B can regulate the differentiation of CD14+ monocytes into mature dendritic cells, thus inhibiting the proliferation of CD4+ T cells and promoting the secretion of IL-4. This suggests that TNFRSF $6 B$ may be an effector molecule capable of directing the $\mathrm{T}$ cell response to the $\mathrm{T}$ helper type 2 (Th2) phenotype (45).

Gene chip detection was performed to analyze the downstream genes of TNFRSF $6 B$ and predict the networks regulated by TNFRSF6B. The results were similar to those predicted by the TCGA and ONCOMINE databases. Interestingly, IPA demonstrated that CEACAM1 plays an important role in the regulatory network of TNFRSF $6 B$.
CEACAM1 is a transmembrane glycoprotein located on the cell surface. It is a member of the carcinoembryonic antigen family, which belongs to the immunoglobulin superfamily adhesion molecules (46). Previous studies have shown that CEACAM1 can promote a variety of malignant tumors such as non-small cell lung cancer (47), colon cancer (48), and pancreatic cancer (49). Possible mechanisms include the prevention of $\mathrm{T}$ lymphocyte activation, stimulation of B lymphocyte proliferation, inhibition of $\mathrm{T}$ cell and NK cell cytotoxicity, promotion of tumor cells invasion, enhancement of endothelial cell viability, promotion of blood vessel formation, and regulation of vascular remodeling $(48,50)$. Therefore, CEACAM1 has many important biological functions. The positive correlation between CEACAM1 and TNFRSF6B in pancreatic cancer cells suggested that the mechanism of TNFRSF $6 B$ may involve inhibiting the activation and proliferation of $\mathrm{T}$ lymphocytes, thereby promoting the rapid proliferation of cancer cells. Therapies which target these two collaborative genes may be an efficient way to treat pancreatic cancer.

In summary, this study demonstrated that TNFRSF $6 B$ is overexpressed in pancreatic cancer tissues compared to healthy pancreatic tissues. Furthermore, elevated TNFRSF $6 B$ expression can promote the growth and invasion of tumor cells, and is related to the OS of patients with pancreatic cancer. In addition, this report demonstrated that TNFRSF6B is widely involved in the regulation of the tumor necrosis factor signaling pathway, the p53 signaling pathway, response to type I interferons, the apoptosis signaling pathway, the immune response, the regulation of immune processes, cell proliferation, the cell cycle, and apoptosis. Therefore, this investigation revealed novel insights into the molecular mechanisms underlying the regulation of TNFRSF6B in pancreatic cancer cells and proposed that TNFRSF6B may be a promising molecular target for the diagnosis and treatment of patients with pancreatic cancer.

\section{Acknowledgments}

Funding: This study was funded by the Project of the National Natural Science Foundation of China (grant number: 81902385), the Foundation Research Project of the Natural Science Foundation of Jiangsu Province (grant number: BK20201173), the Jiangsu Provincial Medical Youth Talent (grant number: QNRC2016734), and the Project of Medical Research of Jiangsu Province (grant number: Y2018094). 


\section{Footnote}

Reporting Checklist: The authors have completed the MDAR checklist. Available at https://dx.doi.org/10.21037/jgo-21-303

Conflicts of Interest: All authors have completed the ICMJE uniform disclosure form (available at https://dx.doi. org/10.21037/jgo-21-303). The authors have no conflicts of interest to declare.

Ethical Statement: The authors are accountable for all aspects of the work in ensuring that questions related to the accuracy or integrity of any part of the work are appropriately investigated and resolved. The study was conducted in accordance with the Declaration of Helsinki (as revised in 2013). Since all data were extracted from publicly available database, approval by the ethics committee and patient consent were waived.

Open Access Statement: This is an Open Access article distributed in accordance with the Creative Commons Attribution-NonCommercial-NoDerivs 4.0 International License (CC BY-NC-ND 4.0), which permits the noncommercial replication and distribution of the article with the strict proviso that no changes or edits are made and the original work is properly cited (including links to both the formal publication through the relevant DOI and the license). See: https://creativecommons.org/licenses/by-nc-nd/4.0/.

\section{References}

1. Bray F, Ferlay J, Soerjomataram I, et al. Global cancer statistics 2018: GLOBOCAN estimates of incidence and mortality worldwide for 36 cancers in 185 countries. CA Cancer J Clin 2018;68:394-424.

2. Ansari D, Tingstedt B, Andersson B, et al. Pancreatic cancer: yesterday, today and tomorrow. Future Oncol 2016;12:1929-46.

3. Karagiannis GS, Pavlou MP, Diamandis EP. Cancer secretomics reveal pathophysiological pathways in cancer molecular oncology. Mol Oncol 2010;4:496-510.

4. Schaaij-Visser TB, de Wit M, Lam SW, et al. The cancer secretome, current status and opportunities in the lung, breast and colorectal cancer context. Biochim Biophys Acta 2013;1834:2242-58.

5. Villarreal L, Méndez O, Salvans C, et al. Unconventional secretion is a major contributor of cancer cell line secretomes. Mol Cell Proteomics 2013;12:1046-60.
6. Mustafa S, Pan L, Marzoq A, et al. Comparison of the tumor cell secretome and patient sera for an accurate serum-based diagnosis of pancreatic ductal adenocarcinoma. Oncotarget 2017;8:11963-76.

7. Grønborg M, Kristiansen TZ, Iwahori A, et al. Biomarker discovery from pancreatic cancer secretome using a differential proteomic approach. Mol Cell Proteomics 2006;5:157-71.

8. Pitti RM, Marsters SA, Lawrence DA, et al. Genomic amplification of a decoy receptor for Fas ligand in lung and colon cancer. Nature 1998;396:699-703.

9. Zhao T, Xu Y, Ren S, et al. The siRNA silencing of DcR3 expression induces Fas ligand-mediated apoptosis in HepG2 cells. Exp Ther Med 2018;15:4370-8.

10. Ma Z, Wang B, Wang M, et al. TL1A increased IL-6 production on fibroblast-like synoviocytes by preferentially activating TNF receptor 2 in rheumatoid arthritis. Cytokine 2016;83:92-8.

11. del Rio ML, Fernandez-Renedo C, Chaloin O, et al. Immunotherapeutic targeting of LIGHT/LT R/ HVEM pathway fully recapitulates the reduced cytotoxic phenotype of LIGHT-deficient T cells. MAbs 2016;8:478-90.

12. Zhang Y, Luo J, He R, et al. Expression and clinicopathological implication of DcR3 in lung cancer tissues: a tissue microarray study with 365 cases. Onco Targets Ther 2016;9:4959-68.

13. Hu R, Liu W, Qiu X, et al. Expression of tumor necrosis factor- $\alpha$-induced protein 8 in stage III gastric cancer and the correlation with DcR3 and ERK1/2. Oncol Lett 2016;11:1835-40.

14. Ge H, Liang C, Li Z, et al. DcR3 induces proliferation, migration, invasion, and EMT in gastric cancer cells via the PI3K/AKT/GSK-3 $\beta / \beta$-catenin signaling pathway. Onco Targets Ther 2018;11:4177-87.

15. Chen J, Guo XZ, Li HY, et al. Dendritic cells engineered to secrete anti-DcR3 antibody augment cytotoxic $\mathrm{T}$ lymphocyte response against pancreatic cancer in vitro. World J Gastroenterol 2017;23:817-29.

16. Wang W, Zhang M, Sun W, et al. Reduction of decoy receptor 3 enhances TRAIL-mediated apoptosis in pancreatic cancer. PLoS One 2013;8:e74272.

17. Yu W, Xu YC, Tao Y, et al. DcR3 regulates the growth and metastatic potential of SW480 colon cancer cells. Oncol Rep 2013;30:2741-8.

18. Liang QL, Wang BR, Li GH. DcR3 and survivin are highly expressed in colorectal carcinoma and closely correlated to its clinicopathologic parameters. J Zhejiang 
Univ Sci B 2009;10:675-82.

19. Jiang $M$, Lin $X, H e R$, et al. Decoy Receptor 3 (DcR3) as a Biomarker of Tumor Deterioration in Female Reproductive Cancers: A Meta-Analysis. Med Sci Monit 2016;22:1850-7.

20. Wei Y, Chen X, Yang J, et al. DcR3 promotes proliferation and invasion of pancreatic cancer via a DcR3/STAT1/IRF1 feedback loop. Am J Cancer Res 2019;9:2618-33.

21. Rhodes DR, Kalyana-Sundaram S, Mahavisno V, et al. Oncomine 3.0: genes, pathways, and networks in a collection of 18,000 cancer gene expression profiles. Neoplasia 2007;9:166-80.

22. Tang Z, Li C, Kang B, et al. GEPIA: a web server for cancer and normal gene expression profiling and interactive analyses. Nucleic Acids Res 2017;45:W98-102.

23. Li T, Fan J, Wang B, et al. TIMER: A Web Server for Comprehensive Analysis of Tumor-Infiltrating Immune Cells. Cancer Res 2017;77:e108-10.

24. Warde-Farley D, Donaldson SL, Comes O, et al. The GeneMANIA prediction server: biological network integration for gene prioritization and predicting gene function. Nucleic Acids Res 2010;38:W214-20.

25. Li D, Xie K, Wolff R, et al. Pancreatic cancer. Lancet 2004;363:1049-57.

26. Vincent A, Herman J, Schulick R, et al. Pancreatic cancer. Lancet 2011;378:607-20.

27. Sohal DPS, Kennedy EB, Cinar P, et al. Metastatic Pancreatic Cancer: ASCO Guideline Update. J Clin Oncol 2020;JCO2001364.

28. Li H, Zhang L, Lou H, et al. Overexpression of decoy receptor 3 in precancerous lesions and adenocarcinoma of the esophagus. Am J Clin Pathol 2005;124:282-7.

29. Roth W, Isenmann S, Nakamura M, et al. Soluble decoy receptor 3 is expressed by malignant gliomas and suppresses CD95 ligand-induced apoptosis and chemotaxis. Cancer Res 2001;61:2759-65.

30. Arakawa Y, Tachibana O, Hasegawa M, et al. Frequent gene amplification and overexpression of decoy receptor 3 in glioblastoma. Acta Neuropathol 2005;109:294-8.

31. Connor JP, Felder M. Ascites from epithelial ovarian cancer contain high levels of functional decoy receptor 3 (DcR3) and is associated with platinum resistance. Gynecol Oncol 2008;111:330-5.

32. Ohshima K, Haraoka S, Sugihara M, et al. Amplification and expression of a decoy receptor for fas ligand (DcR3) in virus (EBV or HTLV-I) associated lymphomas. Cancer Lett 2000;160:89-97.

33. Chang PM, Chen PM, Hsieh SL, et al. Expression of a soluble decoy receptor 3 in patients with diffuse large B-cell lymphoma predicts clinical outcome. Int J Oncol 2008;33:549-54.

34. Brunetti G, Oranger A, Mori G, et al. The formation of osteoclasts in multiple myeloma bone disease patients involves the secretion of soluble decoy receptor 3 . Ann N Y Acad Sci 2010;1192:298-302.

35. Tu HF, Liu CJ, Liu SY, et al. Serum decoy receptor 3 level: a predictive marker for nodal metastasis and survival among oral cavity cancer patients. Head Neck 2011;33:396-402.

36. Chen G, Luo D. Expression of decoy receptor 3 in liver tissue microarrays. Natl Med J India 2008;21:275-8.

37. Lin WW, Hsieh SL. Decoy receptor 3: a pleiotropic immunomodulator and biomarker for inflammatory diseases, autoimmune diseases and cancer. Biochem Pharmacol 2011;81:838-47.

38. Shi G, Wu Y, Zhang J, et al. Death decoy receptor TR6/ DcR3 inhibits $\mathrm{T}$ cell chemotaxis in vitro and in vivo. $\mathrm{J}$ Immunol 2003;171:3407-14.

39. You RI, Chang YC, Chen PM, et al. Apoptosis of dendritic cells induced by decoy receptor 3 (DcR3). Blood 2008;111:1480-8.

40. Yang CR, Hsieh SL, Teng CM, et al. Soluble decoy receptor 3 induces angiogenesis by neutralization of TL1A, a cytokine belonging to tumor necrosis factor superfamily and exhibiting angiostatic action. Cancer Res 2004;64:1122-9.

41. Chang YC, Hsu TL, Lin HH, et al. Modulation of macrophage differentiation and activation by decoy receptor 3. J Leukoc Biol 2004;75:486-94.

42. Wan X, Shi G, Semenuk M, et al. DcR3/TR6 modulates immune cell interactions. J Cell Biochem 2003;89:603-12.

43. Migone TS, Zhang J, Luo X, et al. TL1A is a TNF-like ligand for DR3 and TR6/DcR3 and functions as a T cell costimulator. Immunity 2002;16:479-92.

44. Hsu TL, Wu YY, Chang YC, et al. Attenuation of Th1 response in decoy receptor 3 transgenic mice. J Immunol 2005;175:5135-45.

45. Hsu TL, Chang YC, Chen SJ, et al. Modulation of dendritic cell differentiation and maturation by decoy receptor 3. J Immunol 2002;168:4846-53.

46. Laurie NA, Comegys MM, Carreiro MP, et al. Carcinoembryonic antigen-related cell adhesion molecule 1a-4L suppression of rat hepatocellular carcinomas. Cancer Res 2005;65:11010-7.

47. Wang L, Lin SH, Wu WG, et al. C-CAM1, a candidate tumor suppressor gene, is abnormally expressed in primary 
lung cancers. Clin Cancer Res 2000;6:2988-93.

48. Leung N, Turbide C, Olson M, et al. Deletion of the carcinoembryonic antigen-related cell adhesion molecule 1 (Ceacam1) gene contributes to colon tumor progression in a murine model of carcinogenesis. Oncogene 2006;25:5527-36.

49. Gebauer F, Wicklein D, Horst J, et al. Carcinoembryonic antigen-related cell adhesion molecules (CEACAM) 1, 5 and 6 as biomarkers in pancreatic cancer. PLoS One 2014;9:e113023.

50. Horst AK, Ito WD, Dabelstein J, et al. Carcinoembryonic antigen-related cell adhesion molecule 1 modulates vascular remodeling in vitro and in vivo. $\mathrm{J}$ Clin Invest 2006;116:1596-605.

Cite this article as: Zhang $\mathrm{C}$, Li H, Huang Y, Tang Y, Wang J, Cheng Y, Wei Y, Zhu D, Cao Z, Zhou J. Integrative analysis of TNFRSF6B as a potential therapeutic target for pancreatic cancer. J Gastrointest Oncol 2021;12(4):1673-1690. doi: 10.21037/ jgo-21-303 
A

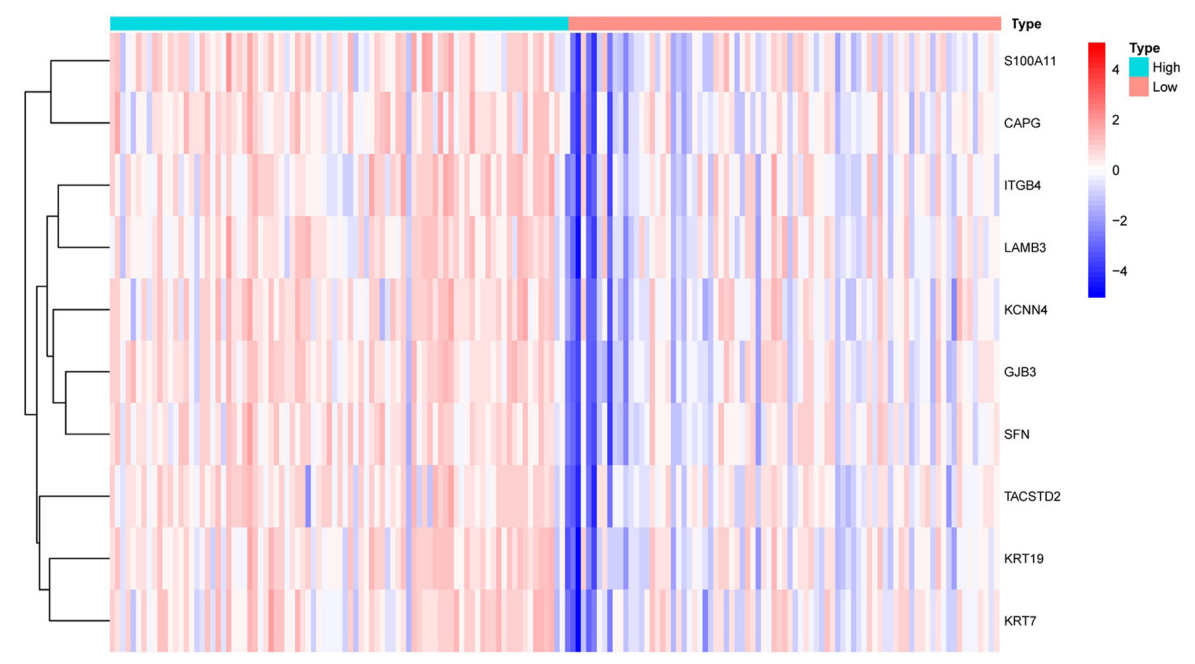

B
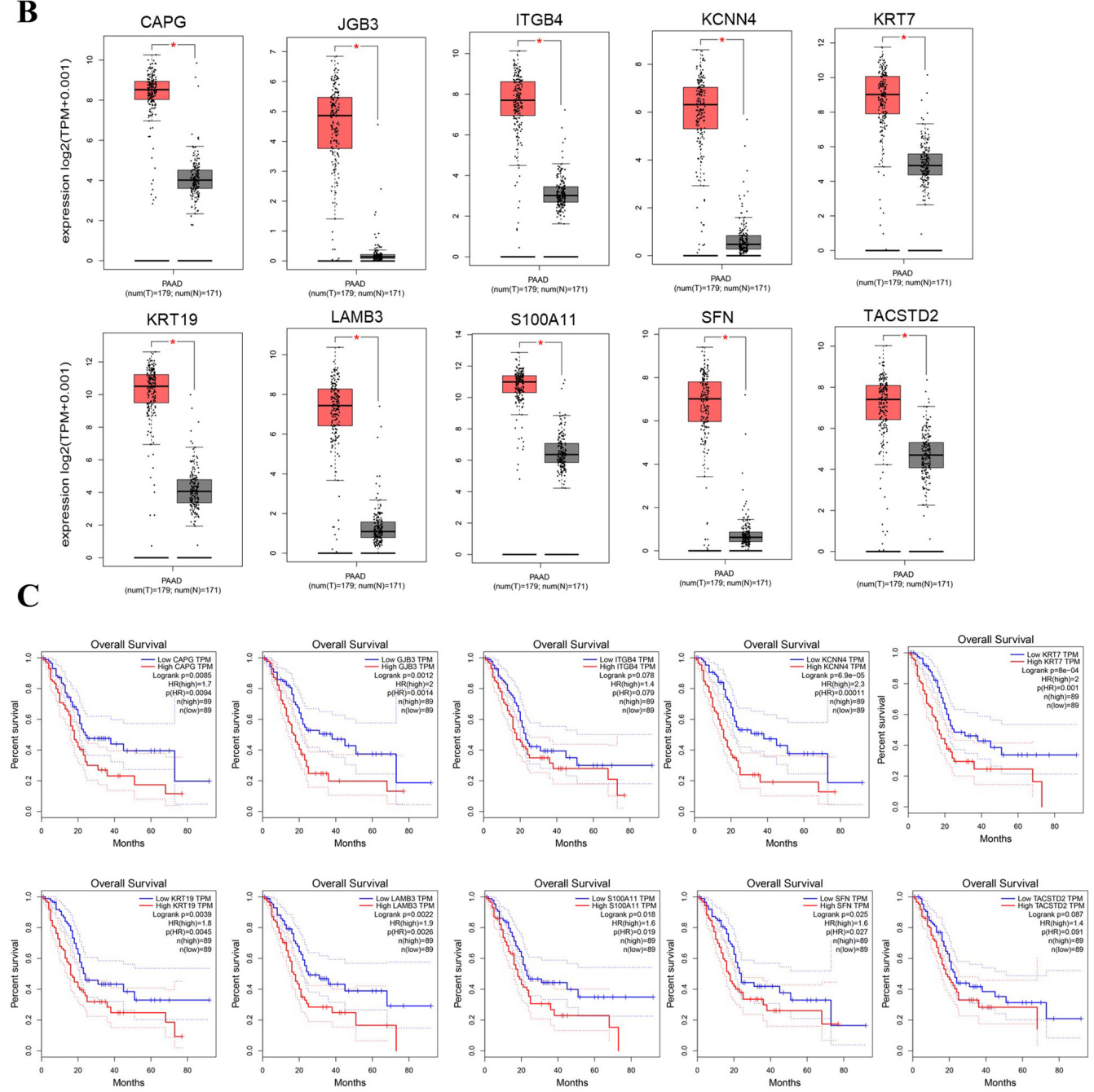

Figure S1 The expression and prognostic value of 10 hub genes in PAAD. (A) Heatmap representation of the correlation between TNFRSF6B expression and 10 hub genes. Red color represents positive correlation and blue color represents negative correlation; (B) the expression of 10 hub genes (S100A11, GJB3, TACSTD2, KRT7, KCNN4, CAPG, ITGB4, LAMB3, SFN, KRT19) in PAAD; (C) the overall survival curve of S100A11, GFB3, TACSTD2, KRT7, KCNN4, CAPG, ITGB4, LAMB3, SFN, KRT19. PAAD, pancreatic adenocarcinoma. 

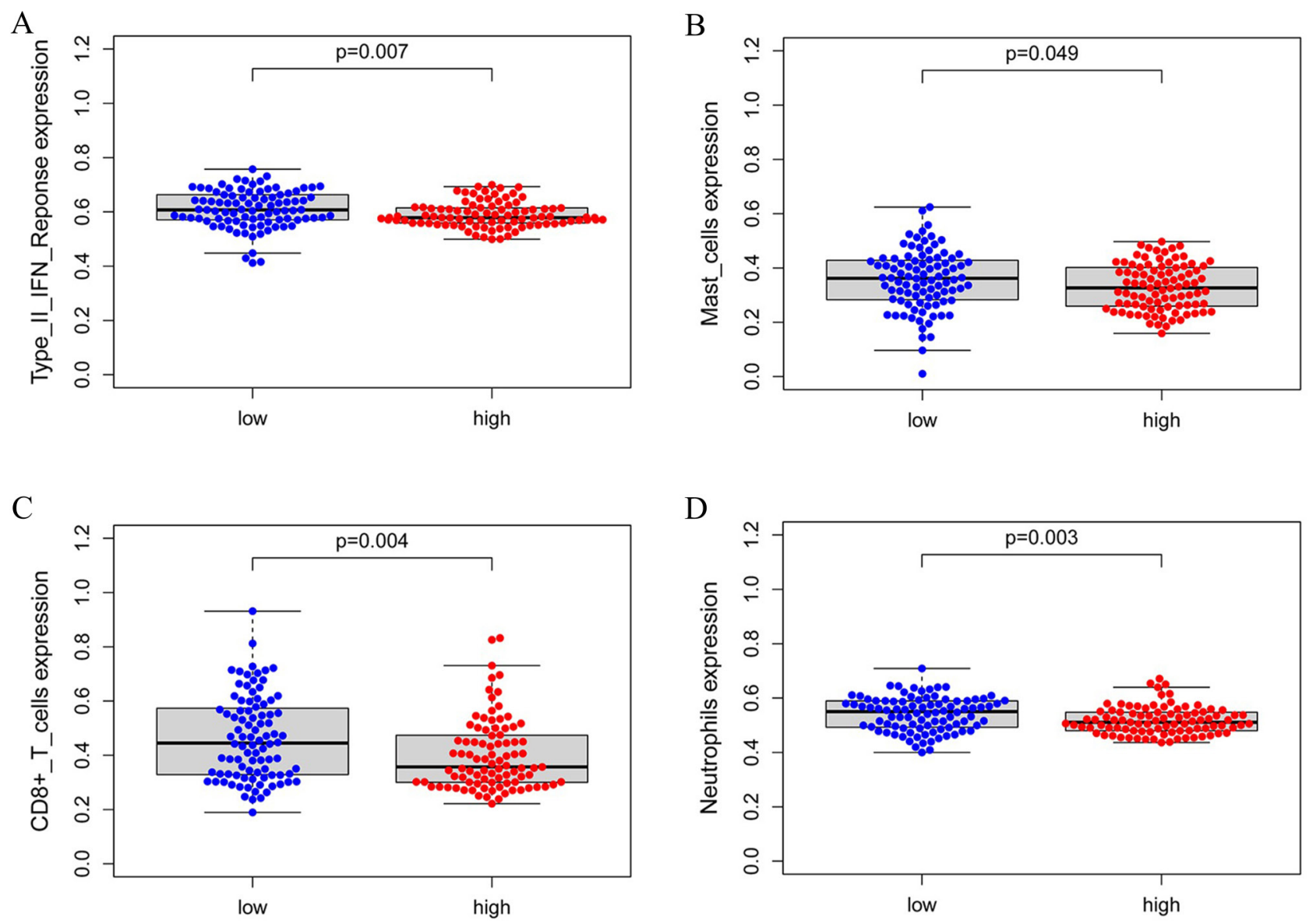

Figure S2 Associations between TNFRSF6B gene expressions and immune cells in PAAD. TNFRSF6B has a negative correlation with TypeII INF-Response (A), mast cells (B), CD8(+) T cell (C) and neutrophils (D). TNFRSF6B, tumor necrosis factor receptor superfamily member 6b; PAAD, pancreatic adenocarcinoma; INF, interferon. 
A
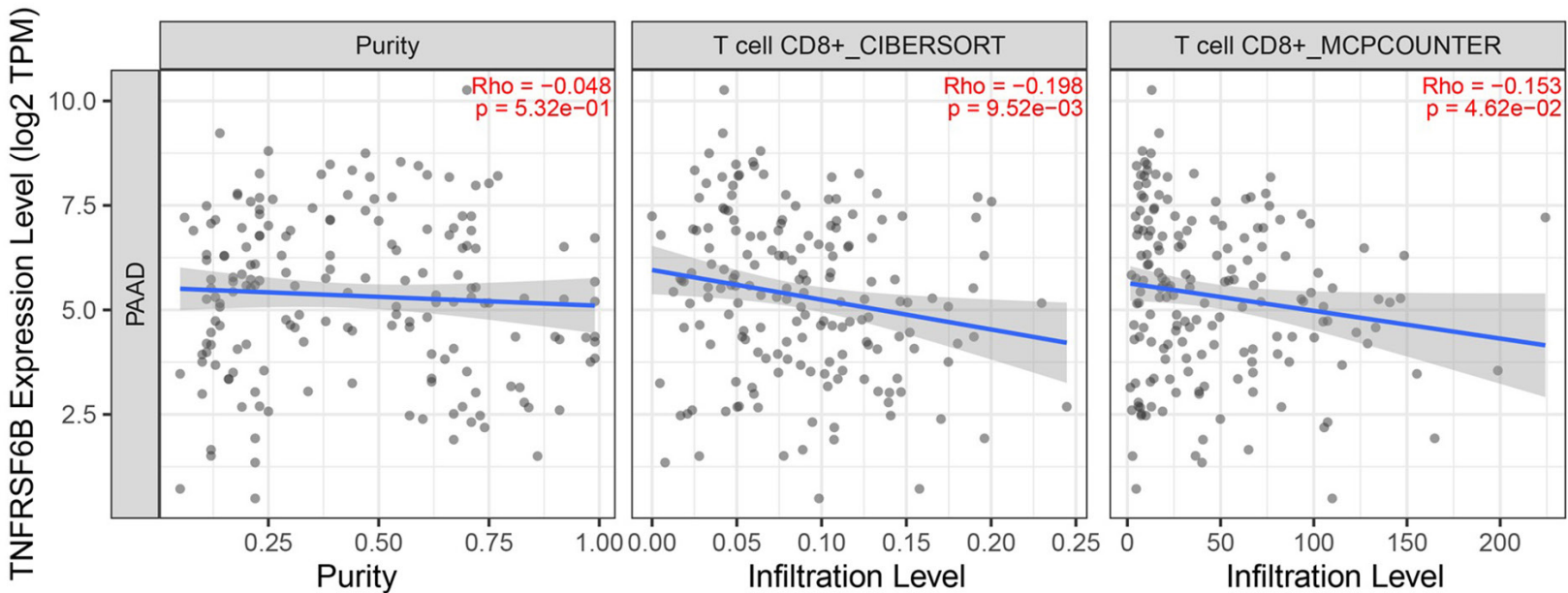

$\mathrm{B}$
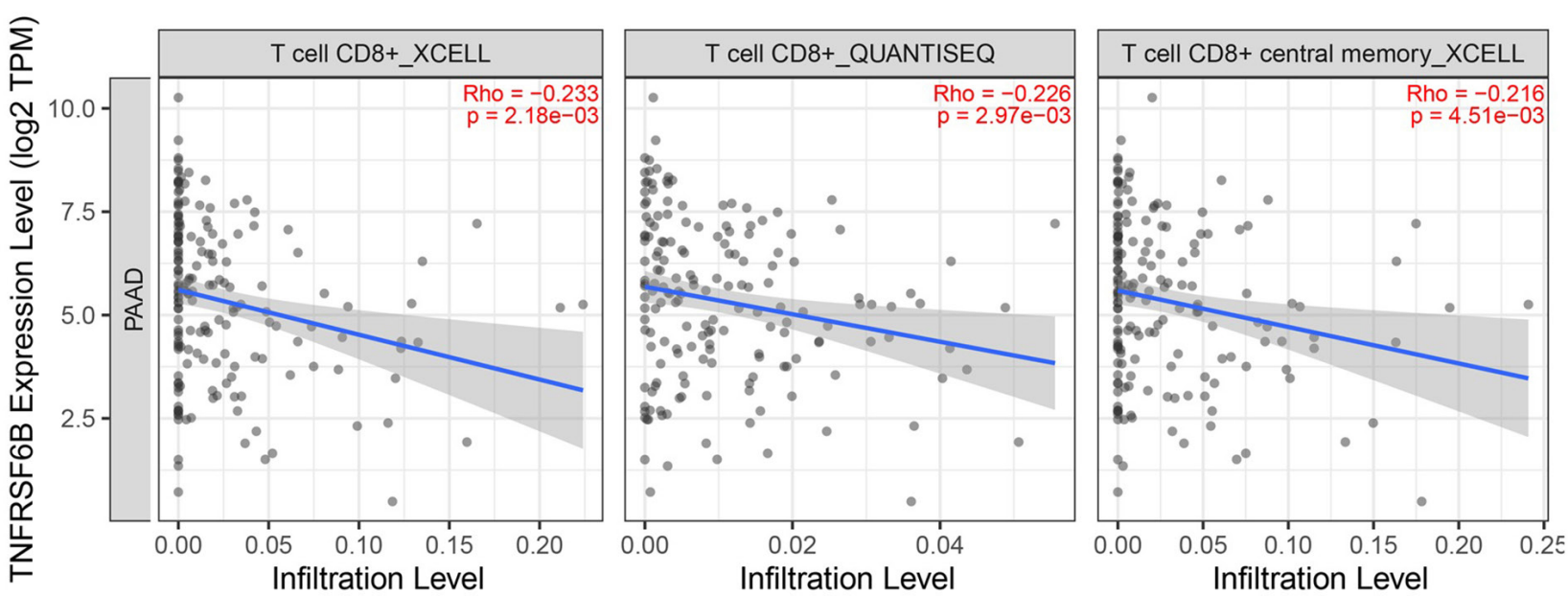

Figure S3 Correlation between TNFRSF6B expression and CD8(+) T cell in PAAD. TNFRSF6B was negatively correlated with CD8(+) T cell. TNFRSF6B, tumor necrosis factor receptor superfamily member 6b; PAAD, pancreatic adenocarcinoma. 